\title{
Function spaces and classifying spaces of algebras over a prop
}

\author{
SINAN YALIN
}

\begin{abstract}
The goal of this paper is to prove that the classifying spaces of categories of algebras governed by a prop can be determined by using function spaces on the category of props. We first consider a function space of props to define the moduli space of algebra structures over this prop on an object of the base category. Then we mainly prove that this moduli space is the homotopy fiber of a forgetful map of classifying spaces, generalizing to the prop setting a theorem of Rezk.
\end{abstract}

The crux of our proof lies in the construction of certain universal diagrams in categories of algebras over a prop. We introduce a general method to carry out such constructions in a functorial way.

18D10, 18D50, 18G55, 55U10

\section{Introduction}

Associative algebras, Lie algebras, Poisson algebras and their variants play a key role in algebra, topology, category theory, differential and algebraic geometry, mathematical physics. They all share the common feature of being defined by operations with several inputs and one single output (the associative product, the Lie bracket, the Poisson bracket). A powerful device to handle such kinds of algebraic structure is the notion of operad, which has proven to be a fundamental tool to study algebras such as the aforementioned examples, feeding back important outcomes in these various fields. However, algebraic structures equipped not only with products but also with coproducts play a crucial role in various places in mathematics. It is worth mentioning, for instance, the following important examples: Hopf algebras in representation theory and mathematical physics, Frobenius algebras encompassing the Poincaré duality phenomenon in algebraic topology (which corresponds to unitary and counitary Frobenius bialgebras, see Kock [16]), Lie bialgebras introduced by Drinfeld in quantum group theory (see Drinfel'd [6; 7]), involutive Lie bialgebras originally encoding operations on free loops on surfaces in the work of Turaev [24] and then generalized to higher dimensional manifolds by Chas and Sullivan [4] in string topology [3]. A convenient way to handle such kinds of structure is to use the formalism of props, a generalization of operads 
encoding algebraic structures based on operations with several inputs and several outputs. A dg prop is a collection of complexes $P=\{P(m, n)\}_{m, n \in \mathbb{N}}$, where each $P(m, n)$ represents formal operations with $m$ inputs and $n$ outputs. This collection $P$ is equipped with composition products grafting and concatenating these operations in a compatible way.

This paper is a follow-up to Yalin [25], where we set up a homotopy theory for algebras over (possibly colored) differential graded (dg for short) props. The crux of our approach lies in the proof that the Dwyer-Kan classifying spaces attached to categories of algebras over dg props are homotopy invariants of the dg prop. Such spaces have been introduced by Dwyer and Kan in their seminal work on simplicial localization of categories; see $[10 ; 8 ; 9]$. Recall from these papers that the classifying space of a category with weak equivalences (for instance a model category) is the nerve of its subcategory of weak equivalences. It encodes information about homotopy types and internal symmetries of the objects, ie their homotopy automorphisms. The goal of the present paper is to give another description of these classifying spaces, in terms of function spaces of dg prop morphisms, in order to make their homotopy theory accessible to computation. These function spaces are moduli spaces of algebra structures, that is, simplicial sets $P\{X\}$ whose vertices are dg prop morphisms $P \rightarrow$ End $_{X}$ representing a $P$-algebra structure on an object $X$ of the base category. For us, the base category is the category $\mathrm{Ch}$ of $\mathbb{Z}$-graded chain complexes over a field $\mathbb{K}$. Let $\mathrm{Ch}^{P}$ be the category of $P$-algebras and $w \mathrm{Ch}^{P}$ be its subcategory obtained by restriction to morphisms which are quasiisomorphisms in $\mathrm{Ch}$. Let us denote by $\mathcal{N}(-)$ the nerve of a category. Our main theorem reads as follows.

Theorem 0.1 Let $P$ be a cofibrant $d g$ prop defined in the category $C h$ of chain complexes and let $X \in \mathcal{C}$. The commutative square

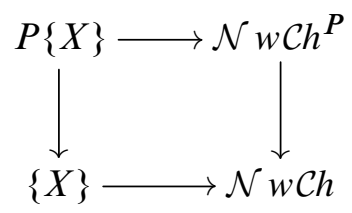

is a homotopy pullback of simplicial sets.

As a consequence, we get the following decomposition of function spaces in terms of homotopy automorphisms.

Corollary 0.2 We have

$$
P\{X\} \sim \coprod_{[X]} L w \mathcal{C h}^{P}(X, X)
$$


where $L(-)$ is the simplicial localization functor of Dwyer-Kan [8], and $[X]$ ranges over the weak equivalence classes of $P$-algebras having $X$ as underlying object. In particular, the simplicial monoids of homotopy automorphisms $\operatorname{LwCh}^{P}(X, X)$ are homotopically small in the sense of Dwyer-Kan, that is, their homotopy groups are all small as sets.

Theorem 0.1 is a broad generalization of the first main result of Rezk's thesis [21, Theorem 1.1.5], which concerns the case of operads in simplicial sets and simplicial modules. However, the method of [21] relies on the existence of a model category structure on algebras over operads, which does not exist anymore for algebras over dg props. The crux of the proof of Theorem 0.1 lies in the construction of functorial diagram factorizations in categories of algebras over dg props. We use a new approach, relying on universal categories of algebras over dg props, to perform such constructions in our context. This method enables us to get around the lack of model structure.

We would like to emphasize some links with two other objects encoding algebraic structures and their deformations. Theorem 0.1 asserts that we can use a function space of dg props, the moduli space $P\{X\}$, to determine classifying spaces of categories of algebras over dg props $\mathcal{N} w \mathrm{Ch}^{P}$. The homotopy groups of this moduli space, in turn, can be approached by means of a Bousfield-Kan-type spectral sequence. The $E_{2}$-page of this spectral sequence is identified with the cohomology of certain deformation complexes. These complexes have been studied in Frégier, Markl and Yau [11], Markl [18] and Merkulov and Vallette [19]. These papers prove the existence of an $L_{\infty}$-structure on such complexes which generalizes the intrinsic Lie bracket of Schlessinger and Stasheff [23]. We aim to apply this spectral sequence technique and provide new results about the deformation theory of bialgebras in an ongoing work. To complete this outlook, we point out that Ciocan-Fontanine and Kapranov in [5] used a similar approach to that of Rezk in order to define a derived moduli space of algebras structures in the formalism of dg schemes. The author recently proved in [27], by different methods, that the simplicial moduli spaces considered in the present paper are also the global points of derived moduli stacks in the setting of Toën and Vezzosi's derived algebraic geometry, and that the deformation complexes of [19] really compute the tangent complexes of these stacks.

Organization In Section 1, we briefly recall several properties of dg props and their algebras, and we define the notion of moduli space of algebraic structures. In Section 2, we revisit the notion of a colored dg prop as a symmetric monoidal dg category generated by words of colors. Then we carry out the main technical construction of this section: a dg category associated to the data of a small category $\mathcal{J}$ and a colored dg prop $P_{\mathcal{I}}$ encoding $\mathcal{I}$-diagrams of $P$-algebras, where $\mathcal{I}$ is a subcategory of $\mathcal{J}$. This category 
of "formal variables" is used to explain how a functorial $\mathcal{I}$-diagram of $P$-algebras can be extended to a functorial $\mathcal{J}$-diagram of $P$-algebras under several technical assumptions. This construction applies in particular to the functorial factorizations of morphisms provided by the axioms of model categories. In Section 3, we prove that the classifying space of quasi-isomorphisms of $P$-algebras is weakly equivalent to the classifying space of acyclic surjections of $P$-algebras. For this, we need to examine in Section 3 how the internal and external tensor products of a diagram category behave with respect to its injective and projective model structures. The projective case is more subtle and does not appear in the literature. Then we combine the results of Section 2, those of Section 3 and Quillen's Theorem A to provide this weak equivalence (induced by an inclusion of categories). Finally, in Section 4 we rely on the previous results to generalize [21, Theorem 1.1.5] to the dg prop setting.

Acknowledgements I would like to thank Benoit Fresse for his useful remarks. I also thank the referee for careful reading and useful comments.

\section{Props, algebras and moduli spaces}

Throughout this paper, we work in the category $\mathcal{C} h$ of $\mathbb{Z}$-graded chain complexes over a field $\mathbb{K}$. We write "dg" as an abbreviation for "differential graded". We briefly recall our conventions and the main definitions concerning $\mathrm{dg}$ props in this section. We refer to [12] for a more comprehensive account.

\subsection{Background on props and their algebras}

An $\mathbb{S}$-biobject in $\mathcal{C} h$ is a double sequence $\{M(m, n) \in \mathcal{C} h\}_{(m, n) \in \mathbb{N}^{2}}$, where each $M(m, n)$ is equipped with a right action of the symmetric group on $m$ letters $\Sigma_{m}$, a left action of the symmetric group on $n$ letters $\Sigma_{n}$, such that these actions commute with each other.

Definition 1.1 A dg prop is an $\mathbb{S}$-biobject in $\mathcal{C}$ endowed with associative horizontal composition products

$$
{ }_{h}: P\left(m_{1}, n_{1}\right) \otimes P\left(m_{2}, n_{2}\right) \rightarrow P\left(m_{1}+m_{2}, n_{1}+n_{2}\right),
$$

vertical associative composition products

$$
\circ_{v}: P(k, n) \otimes P(m, k) \rightarrow P(m, n)
$$

and units $\eta: \mathbb{K} \rightarrow P(n, n)$. These products satisfy the exchange law

$$
\left(f_{1} \circ_{h} f_{2}\right) \circ_{v}\left(g_{1} \circ_{h} g_{2}\right)=(-1)^{\left|g_{1}\right|\left|f_{2}\right|}\left(f_{1} \circ_{v} g_{1}\right) \circ_{h}\left(f_{2} \circ_{v} g_{2}\right)
$$


and are compatible with the actions of symmetric groups and with the differentials. Morphisms of dg props are equivariant morphisms of collections compatible with the composition products. We denote by Prop the category of dg props.

The following definition shows how a given dg prop encodes algebraic operations on the tensor powers of a chain complex.

Definition 1.2 (1) The endomorphism dg prop of a chain complex $X$ is given by $\operatorname{End}_{X}(m, n)=\operatorname{Hom}_{\mathcal{C} h}\left(X^{\otimes m}, X^{\otimes n}\right)$, where $\operatorname{Hom}_{\mathcal{C} h}(-,-)$ is the internal hom bifunctor of $\mathrm{Ch}$. The horizontal composition is given by the tensor product of homomorphisms and the vertical composition is given by the composition of homomorphisms.

(2) Let $P$ be a dg prop. A $P$-algebra is a chain complex $X$ equipped with a dg prop morphism $P \rightarrow$ End $_{X}$.

Hence any "abstract" operation of $P$ is sent to an operation on $X$, and the way abstract operations compose under the composition products of $P$ tells us the relations satisfied by the corresponding algebraic operations on $X$.

One can carry out similar constructions in the category of colored $\mathbb{S}$-biobjects in order to define colored dg props and their algebras.

Definition 1.3 Let $C$ be a non-empty set, called the set of colors.

(1) A $C$-colored $\mathbb{S}$-biobject $M$ is a double sequence of chain complexes

$$
\{M(m, n)\}_{(m, n) \in \mathbb{N}^{2}},
$$

where each $M(m, n)$ admits commuting right $\Sigma_{m}$ action and left $\Sigma_{n}$ action as well as a decomposition

$$
M(m, n)=\bigoplus_{c_{i}, d_{i} \in C} M\left(c_{1}, \ldots, c_{m} ; d_{1}, \ldots, d_{n}\right)
$$

compatible with these actions. The objects $M\left(c_{1}, \ldots, c_{m} ; d_{1}, \ldots, d_{n}\right)$ should be thought of as spaces of operations with colors $c_{1}, \ldots, c_{m}$ indexing the $m$ inputs and colors $d_{1}, \ldots, d_{n}$ indexing the $n$ outputs.

(2) A $C$-colored dg prop $P$ is a $C$-colored $\mathbb{S}$-biobject endowed with a horizontal composition

$$
\begin{aligned}
\circ_{h}: P\left(c_{11}, \ldots, c_{1 m_{1}} ; d_{11}, \ldots, d_{1 n_{1}}\right) \otimes \cdots \otimes P\left(c_{k 1}, \ldots, c_{k m_{k}} ; d_{k 1}, \ldots, d_{k n_{1}}\right) \\
\rightarrow P\left(c_{11}, \ldots, c_{k m_{k}} ; d_{k 1}, \ldots, d_{k n_{k}}\right) \subseteq P\left(m_{1}+\cdots+m_{k}, n_{1}+\cdots+n_{k}\right)
\end{aligned}
$$


and a vertical composition

$$
\begin{aligned}
\circ_{v}: P\left(c_{1}, \ldots, c_{k} ; d_{1}, \ldots, d_{n}\right) \otimes P\left(a_{1}, \ldots,\right. & \left.a_{m} ; b_{1}, \ldots, b_{k}\right) \\
& \rightarrow P\left(a_{1}, \ldots, a_{m} ; d_{1}, \ldots, d_{n}\right) \subseteq P(m, n),
\end{aligned}
$$

which is equal to zero unless $b_{i}=c_{i}$ for $1 \leq i \leq k$. These two compositions satisfy associativity axioms (we refer the reader to [14] for details).

Definition 1.4 (1) Let $\left\{X_{c}\right\}_{C}$ be a collection of chain complexes. The $C$-colored endomorphism dg prop $\operatorname{End}_{\left\{X_{c}\right\}_{C}}$ is defined by

$\operatorname{End}_{\left\{X_{c}\right\}_{C}}\left(c_{1}, \ldots, c_{m} ; d_{1}, \ldots, d_{n}\right)=\operatorname{Hom}_{C h}\left(X_{c_{1}} \otimes \cdots \otimes X_{c_{m}}, X_{d_{1}} \otimes \cdots \otimes X_{d_{n}}\right)$.

(2) Let $P$ be a $C$-colored dg prop. A $P$-algebra is the data of a collection of objects $\left\{X_{c}\right\}_{C}$ and a $C$-colored dg prop morphism $P \rightarrow \operatorname{End}\left\{X_{c}\right\}_{C}$.

Remark 1.5 Let $\mathcal{I}$ be a small category and let $P$ be a dg prop. We can build an ob(I)colored dg prop $P_{\mathcal{I}}$ such that the $P_{\mathcal{I}}$-algebras are the $\mathcal{I}$-diagrams of $P$-algebras in $C h$ in the same way as in [17]. We refer the reader to Definition 2.3 where this construction is made explicit.

We provide $\mathrm{Ch}$ with the model category structure such that the weak equivalences are quasi-isomorphisms and fibrations are degreewise surjections. The category of $\mathbb{S}$-biobjects is a diagram category over $\mathcal{C h}$, so it inherits a cofibrantly generated model category structure in which weak equivalences and fibrations are defined componentwise. The free dg prop functor [12, Appendix A] gives rise to an adjunction $\mathrm{Ch}^{\mathbb{S}} \rightleftarrows$ Prop between the category of $\mathbb{S}$-biobjects $\mathrm{Ch}^{\mathbb{S}}$ and the category of dg props Prop, which transfers this model category structure to the category of dg props.

Theorem 1.6 (see [12, Theorem 4.9] and [14, Theorem 1.1]) (1) Suppose that $\operatorname{char}(\mathbb{K})>0$. The category Prop 0 of $d g$ props with non-empty inputs (or outputs) equipped with the classes of componentwise weak equivalences and componentwise fibrations forms a cofibrantly generated semi-model category.

(2) Suppose that char $(\mathbb{K})=0$. Then the entire category of dg props inherits a cofibrantly generated model category structure with the weak equivalences and fibrations as above.

(3) Suppose that $\operatorname{char}(\mathbb{K})=0$. Let $C$ be a non-empty set. Then the category Prop $_{C}$ of $C$-colored dg props forms a cofibrantly generated model category with fibrations and weak equivalences defined componentwise. 
A semi-model category structure is a slightly weakened version of a model category structure where the lifting axioms only work for cofibrations with cofibrant domain, and where the factorization axioms only work for a map with a cofibrant domain (see the relevant section of [12]). The notion of a semi-model category is sufficient to apply the usual constructions of homotopical algebra. A dg prop $P$ has non-empty inputs if it satisfies

$$
P(0, n)= \begin{cases}\mathbb{K} & \text { if } n=0, \\ 0 & \text { otherwise. }\end{cases}
$$

We define in a symmetric way a dg prop with non-empty outputs. Such dg props usually encode algebraic structures without unit or without counit, for instance Lie bialgebras.

We will use all the time the existence of a (semi)-model category structure on dg props. Our results hold over a field of any characteristic: we can work alternatively with every dg prop in characteristic zero or with dg props with non-empty inputs/outputs in positive characteristic.

Finally, we recall from [12] the construction of the endomorphism dg prop associated to a diagram $F: \mathcal{J} \rightarrow \mathcal{C h}$,

$$
\operatorname{End}_{F}(m, n):=\int_{i \in \mathcal{J}} \operatorname{Hom}_{\mathcal{C} h}\left(X_{i}^{\otimes m}, X_{i}^{\otimes n}\right),
$$

where $X_{i}=F(i)$. This end can equivalently be defined as a coreflexive equalizer

$$
\operatorname{End}_{F}(m, n) \longrightarrow \prod_{i \in \mathcal{J}} \operatorname{Hom}_{\mathcal{C} h}\left(X_{i}^{\otimes m}, X_{i}^{\otimes n}\right) \underset{d_{1}}{\stackrel{d_{0}}{\longrightarrow}} \prod_{u: i \rightarrow j} \operatorname{Hom}_{\mathcal{C} h}\left(X_{i}^{\otimes m}, X_{j}^{\otimes n}\right)
$$

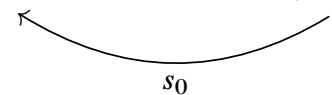

where $d_{0}$ is the product of the maps

$$
u_{*}=\left(F(u)^{\otimes n} \circ-\right): \operatorname{Hom}_{\mathcal{C} h}\left(X_{i}^{\otimes m}, X_{i}^{\otimes n}\right) \rightarrow \operatorname{Hom}_{\mathcal{C} h}\left(X_{i}^{\otimes m}, X_{j}^{\otimes n}\right)
$$

induced by the morphisms $u: i \rightarrow j$ of $\mathcal{J}$, and $d_{1}$ is the product of the maps

$$
u^{*}=\left(-\circ F(u)^{\otimes m}\right): \operatorname{Hom}_{\mathcal{C} h}\left(X_{j}^{\otimes m}, X_{j}^{\otimes n}\right) \rightarrow \operatorname{Hom}_{\mathcal{C h}}\left(X_{i}^{\otimes m}, X_{j}^{\otimes n}\right) .
$$

The section $s_{0}$ is the projection on the factors associated to the identities id: $i \rightarrow i$. This construction allows us to characterize a diagram of $P$-algebras $F: \mathcal{J} \rightarrow \mathcal{C} h^{P}$, where $\mathcal{C} h^{P}$ is the category of $P$-algebras in chain complexes, as a dg prop morphism

$$
P \rightarrow \operatorname{End}_{U(F)},
$$

where $U(F)$ is the diagram of chain complexes underlying $F$. 


\subsection{Moduli spaces of algebra structures}

Throughout the text, we use the Kan-Quillen model category structure on simplicial sets. A moduli space of algebra structures over a dg prop $P$, on a given chain complex $X$, is a simplicial set whose points are the dg prop morphisms $P \rightarrow \operatorname{End}_{X}$ and connected components are homotopy classes of $P$-algebra structures on $X$. Such a moduli space can be more generally defined on diagrams of chain complexes. We then deal with endomorphism dg props of diagrams. To properly construct such a simplicial set and give its first fundamental properties, we have to recall some results about cosimplicial and simplicial resolutions in a model category. For the sake of brevity and clarity, we refer the reader to [13, Chapter 16] for a complete treatment of the notions of simplicial resolutions, cosimplicial resolutions and Reedy model categories.

Definition 1.7 Let $\mathcal{M}$ be a model category and let $X$ be an object of $\mathcal{M}$.

(1) A cosimplicial resolution of $X$ is a cofibrant approximation to the constant cosimplicial object $c c_{*} X$ in the Reedy model category structure on cosimplicial objects $\mathcal{M}^{\Delta}$ of $\mathcal{M}$.

(2) A simplicial resolution of $X$ is a fibrant approximation to the constant simplicial object $c s_{*} X$ in the Reedy model category structure on simplicial objects $\mathcal{M}^{\Delta^{\text {op }}}$ of $\mathcal{M}$.

Definition 1.8 Let $\mathcal{M}$ be a model category and let $X$ be an object of $\mathcal{M}$.

(1) A cosimplicial frame on $X$ is a cosimplicial object $\tilde{X}$ in $\mathcal{M}$, together with a weak equivalence $\tilde{X} \rightarrow c c_{*} X$ in the Reedy model category structure of $\mathcal{M}^{\Delta}$. It has to satisfy the two following properties: the induced map $\tilde{X}^{0} \rightarrow X$ is an isomorphism, and if $X$ is cofibrant in $\mathcal{M}$ then $\tilde{X}$ is cofibrant in $\mathcal{M}^{\Delta}$.

(2) A simplicial frame on $X$ is a simplicial object $\tilde{X}$ in $\mathcal{M}$, together with a weak equivalence $c s_{*} X \rightarrow \tilde{X}$ in the Reedy model category structure of $\mathcal{M}^{\Delta}$. It has to satisfy the following two properties: the induced map $X \rightarrow \tilde{X}^{0}$ is an isomorphism, and if $X$ is fibrant in $\mathcal{M}$ then $\tilde{X}$ is fibrant in $\mathcal{M}^{\Delta^{\text {op }}}$.

Proposition 1.9 [13, Proposition 16.1.9] Let $\mathcal{M}$ be a model category. There exist functorial simplicial resolutions and functorial cosimplicial resolutions in $\mathcal{M}$.

Proposition 1.10 [13, Proposition 16.6.3] Let $X$ be an object of $\mathcal{M}$.

(1) If $X$ is cofibrant then every cosimplicial frame of $X$ is a cosimplicial resolution of $X$.

(2) If $X$ is fibrant then every simplicial frame of $X$ is a simplicial resolution of $X$. 
In a model category $\mathcal{M}$, one can define homotopy mapping spaces $\operatorname{Map}_{\mathcal{M}}(-,-)$, which are simplicial sets equipped with a composition law defined up to homotopy. There are two possible definitions. We can take either $\operatorname{Map}_{\mathcal{M}}(X, Y)=\operatorname{Mor}_{\mathcal{M}}\left(X \otimes \Delta^{\bullet}, Y\right)$, where $(-) \otimes \Delta^{\bullet}$ is a cosimplicial resolution, or $\operatorname{Map}_{\mathcal{M}}(X, Y)=\operatorname{Mor}_{\mathcal{M}}\left(X, Y^{\Delta^{\bullet}}\right)$, where $(-)^{\Delta^{\bullet}}$ is a simplicial resolution. When $X$ is cofibrant and $Y$ is fibrant, these two definitions give the same homotopy type of mapping space and have also the homotopy type of Dwyer and Kan's hammock localization $L^{H}(\mathcal{M}, w \mathcal{M})(X, Y)$, where $w \mathcal{M}$ is the subcategory of weak equivalences of $\mathcal{M}$; see [9]. Moreover, the set of connected components $\pi_{0} \operatorname{Map}_{\mathcal{M}}(X, Y)$ is the set of homotopy classes $[X, Y]_{\mathcal{M}}$ in $\operatorname{Ho}(\mathcal{M})$.

Proposition 1.11 [13, Corollaries 16.5.3 and 16.5.4] Let $\mathcal{M}$ be a model category and $C$ a cosimplicial resolution in $\mathcal{M}$.

(1) If $Y$ is a fibrant object of $\mathcal{M}$, then $\operatorname{Mor}_{\mathcal{M}}(C, Y)$ is a fibrant simplicial set.

(2) If $p: X \rightarrow Y$ is a fibration in $\mathcal{M}$, then $p_{*}: \operatorname{Mor}_{\mathcal{M}}(C, X) \rightarrow \operatorname{Mor}_{\mathcal{M}}(C, Y)$ is a fibration of simplicial sets, acyclic if $p$ is so.

(3) If $p: X \stackrel{\sim}{\rightarrow} Y$ is a weak equivalence of fibrant objects in $\mathcal{M}$, then

$$
p_{*}: \operatorname{Mor}_{\mathcal{M}}(C, X) \stackrel{\sim}{\rightarrow} \operatorname{Mor}_{\mathcal{M}}(C, Y)
$$

is a weak equivalence of fibrant simplicial sets.

Definition 1.12 Let $P$ be a cofibrant dg prop in $C$. Let $X$ be a chain complex. The moduli space of $P$-algebra structures on $X$ is the simplicial set defined by

$$
P\{X\}=\operatorname{Mor}_{\text {Prop }}\left(P \otimes \Delta^{\bullet}, \operatorname{End}_{X}\right),
$$

where $(-) \otimes \Delta^{\bullet}$ is a functorial cosimplicial frame on Prop. We get a functor

$$
\text { Prop } \rightarrow \text { sSet }, \quad P \mapsto P\{X\},
$$

where sSet is the category of simplicial sets.

We can already get two interesting properties of these moduli spaces.

Lemma 1.13 Let $P$ be a cofibrant dg prop. For any chain complex $X$, the moduli space $P\{X\}$ is a fibrant simplicial set.

Proof Every chain complex is fibrant, and fibrations of dg props are defined componentwise, so $\operatorname{End}_{X}$ is a fibrant dg prop. Given that $P$ is cofibrant, the mapping space $P\{X\}$ is fibrant. 
In this case, the connected components of this moduli space are exactly the homotopy classes of $P$-algebra structures on $X$.

To conclude, let us note that these moduli spaces are a well defined homotopy invariant of algebraic structures over a given object.

Lemma 1.14 Let $X$ be a chain complex. Every weak equivalence of cofibrant dg props $P \stackrel{\sim}{\rightarrow} Q$ gives rise to a weak equivalence of fibrant simplicial sets

$$
Q\{X\} \stackrel{\sim}{\rightarrow} P\{X\}
$$

Proof Let $\varphi: P \rightarrow Q$ be a weak equivalence of cofibrant dg props. According to [13, Proposition 16.1.24], the map $\varphi$ induces a Reedy weak equivalence of cosimplicial resolutions $P \otimes \Delta^{\bullet} \stackrel{\sim}{\rightarrow} Q \otimes \Delta^{\bullet}$. The dg prop $\operatorname{End}_{X}$ is fibrant, so we conclude by [13, Corollary 16.5.5] that this weak equivalence of cosimplicial resolutions induces a weak equivalence between the corresponding moduli spaces.

Remark 1.15 The reader may have noticed that, using the existence of functorial cosimplicial resolutions, Definition 1.12, Lemma 1.13 and Lemma 1.14 could have been stated without the cofibrancy assumption on $P$. In this case, let

$$
P^{\bullet} \stackrel{\sim}{\rightarrow} c c^{\bullet} P
$$

be such a cosimplicial resolution of a dg prop $P$, and

$$
\widetilde{P\{X\}}=\operatorname{Mor}_{\text {Prop }}\left(P^{\bullet}, \operatorname{End}_{X}\right)
$$

be this alternative construction of the moduli space. Let

$$
P_{\infty} \stackrel{\sim}{\rightarrow} P
$$

be a functorial cofibrant resolution of $P$. Then a cosimplicial frame on $P_{\infty}$ is a cosimplicial resolution of $P_{\infty}$ by Proposition 1.10, hence a cosimplicial resolution of $P$ as well. By [13, Proposition 16.1.13], any two cosimplicial resolutions of a given object are related by a zigzag whose middle object is a fibrant cosimplicial resolution, and by [13, Corollary 16.5.5] a Reedy weak equivalence of cosimplicial resolutions induces a weak equivalence of mapping spaces, hence

$$
\widetilde{P\{X\}}=\operatorname{Mor}_{\text {Prop }}\left(P^{\bullet}, \operatorname{End}_{X}\right) \simeq \operatorname{Mor}_{\text {Prop }}\left(P_{\infty} \otimes \Delta^{\bullet}, \operatorname{End}_{X}\right)=P_{\infty}\{X\} .
$$

Our alternative construction of a moduli space directly from a $\mathrm{dg}$ prop $P$ thus has the homotopy type of the moduli space of homotopy $P$-algebra structures constructed in Definition 1.12 from a cofibrant resolution of $P$. 


\section{Dg categories associated to colored dg props}

\subsection{Colored dg props as symmetric monoidal dg categories}

We revisit the definition of colored dg props by explaining how they can alternatively be defined as symmetric monoidal dg categories "monoidally" generated by the set of colors. We start with two simple examples before explaining the general construction.

Example 2.1 Any dg prop in $\mathrm{Ch}$ can alternatively be defined as a dg monoidal category $\operatorname{cat}(P)$ such that $\operatorname{ob}(\operatorname{cat}(P))=\left\{x^{\otimes n}, n \in \mathbb{N}\right\}$ (where $x$ is a formal variable), the tensor product is given by $x^{\otimes m} \otimes x^{\otimes n}=x^{\otimes(m+n)}$ and the complexes of morphisms by

$$
\operatorname{Hom}_{\mathrm{cat}(P)}\left(x^{\otimes m}, x^{\otimes n}\right)=P(m, n) .
$$

The category of $P$-algebras consists of enriched symmetric monoidal functors

$$
\operatorname{cat}(P) \rightarrow \mathcal{C h}
$$

with their natural transformations.

Example 2.2 Let $P$ be a (1-colored) dg prop. There exists a 2-colored dg prop $P_{x \rightarrow y}$ such that the category of $P_{x \rightarrow y}$-algebras is the category of morphisms $f: X \rightarrow Y$ in the category of $P$-algebras $\mathcal{C} h^{P}$. It has two colors $x, y$ and it is generated for the composition products by $P(x, \ldots, x ; x, \ldots, x)=P(m, n)$, by $P(y, \ldots, y ; y, \ldots, y)=$ $P(m, n)$, and by an element $f \in P(x, y)$ which represents an abstract arrow $f: x \rightarrow y$. The associated dg monoidal category $\operatorname{cat}\left(P_{x \rightarrow y}\right)$ is defined in the following way. Let Free $_{\text {mon }}(x, y)$ be the monoid freely generated by the two generators $x$ and $y$, ie the set of words in two letters $w \in \operatorname{Free}_{\text {mon }}(x, y)$. Then the objects of $\operatorname{cat}\left(P_{x \rightarrow y}\right)$ are the "monoidal words"

$$
\mathrm{ob}\left(\operatorname{cat}\left(P_{x \rightarrow y}\right)\right)=\left\{w_{\otimes}(x, y), w \in \operatorname{Free}_{\operatorname{mon}}(x, y)\right\},
$$

where $w_{\otimes}(x, y)$ is the formal tensor product corresponding to the word $w$. The complexes of morphisms are

$$
\operatorname{Hom}_{\mathrm{cat}\left(\boldsymbol{P}_{x \rightarrow y}\right)}\left(w_{\otimes}(x, y), v_{\otimes}(x, y)\right)=P_{x \rightarrow y}(\underline{w}, \underline{v})
$$

where $\underline{w}$ is the ordered sequence of letters, ie colors, appearing in the word $w$. Algebras over $P_{x \rightarrow y}$ are enriched symmetric monoidal functors $\operatorname{cat}\left(P_{x \rightarrow y}\right) \rightarrow \mathrm{Ch}$. A $P_{x \rightarrow y}$ algebra is equivalent to a diagram of $P$-algebras $\{\bullet \rightarrow \bullet\} \rightarrow \mathcal{C} h^{P}$.

These constructions can be generalized to arbitrary diagrams as follows. 
Definition 2.3 Let $\mathcal{I}$ be a small category. Then there exists an $\mathrm{ob}(\mathcal{I})$-colored $\mathrm{dg}$ prop $P_{\mathcal{I}}$ consisting of abstract objects $x_{i}$ associated to $i \in \mathcal{I}$, and the morphisms of $P_{\mathcal{I}}$ are generated by operations $p \in P_{\mathcal{I}}\left(x_{i}^{\otimes m}, x_{i}^{\otimes n}\right)$ associated to each $p \in P(m, n)$ and each variable $x_{i}$, as well as abstract arrows $f: x_{i} \rightarrow x_{j}$ associated to the morphisms of $\mathcal{I}$. The corresponding dg monoidal category cat $\left(P_{\mathcal{I}}\right)$ is defined as follows:

$$
\operatorname{ob}\left(\operatorname{cat}\left(P_{\mathcal{I}}\right)\right)=\left\{w_{\otimes}\left(x_{i}, i \in \mathrm{ob}(\mathcal{I})\right), w \in \operatorname{Free}_{\operatorname{mon}}\left(x_{i}, i \in \mathrm{ob}(\mathcal{I})\right)\right\} .
$$

The tensor product is defined by

$$
w_{\otimes}\left(x_{i}, i \in \mathrm{ob}(\mathcal{I})\right) \otimes v_{\otimes}\left(x_{i}, i \in \mathrm{ob}(\mathcal{I})\right)=(w * v)_{\otimes}\left(x_{i}, i \in \mathrm{ob}(\mathcal{I})\right) .
$$

The complexes of morphisms are

$$
\operatorname{Hom}_{\mathrm{cat}\left(\boldsymbol{P}_{\mathcal{I}}\right)}\left(w_{\otimes}\left(x_{i}, i \in \mathrm{ob}(\mathcal{I})\right), v_{\otimes}\left(x_{i}, i \in \mathrm{ob}(\mathcal{I})\right)\right)=P_{\mathcal{I}}(\underline{w}, \underline{v}) .
$$

The composition on the dg hom is the vertical composition product of $P_{\mathcal{I}}$, and the tensor product of morphisms is the horizontal composition product of $P_{\mathcal{I}}$.

In other words, the category $\operatorname{cat}\left(P_{\mathcal{I}}\right)$ is a differential graded monoidal category monoidally generated on objects by the set of colors of $P_{\mathcal{I}}$. This can be generalized in any symmetric monoidal category, giving an alternative definition of a colored dg prop.

Definition 2.4 (1) A $C$-colored dg prop is a small symmetric monoidal dg category monoidally generated by $C$.

(2) A $P_{\mathcal{I}}$-algebra is a symmetric monoidal dg functor $\operatorname{cat}\left(P_{\mathcal{I}}\right) \rightarrow \mathcal{C h}$.

Proposition 2.5 A $P_{\mathcal{I}}$-algebra corresponds to an $\mathcal{I}$-diagram of $P$-algebras.

This result follows from the construction of $P_{\mathcal{I}}$ in terms of generators and relations. For more details we refer the reader to [17, Section 2], where such a construction is carried out in the case of colored dg operads.

\subsection{Categories of universal twisted sums and functorial diagrams of algebras}

Let $P_{\mathcal{J}}$ be a colored prop on a small category $\mathcal{J}$. The category cat $\left(P_{\mathcal{J}}\right)$ reflects the universal structures of the symmetric monoidal category defined by a $P$-algebra in the category of chain complexes. But for some constructions of homotopy theory, we need operations of the ambient category of chain complexes which lie outside the image of this category cat $\left(P_{\mathcal{J}}\right)$. Namely, we need to perform direct sums $C \oplus D$, suspensions $\Sigma C$, and twisted complexes $(C, d)$ which we form by adding a twisting homomorphism 
$d \in \operatorname{Hom}(C, C)$ to the internal differential of a chain complex $\delta: C \rightarrow C$. These operations can clearly not be formed within the image of $\operatorname{cat}\left(P_{\mathcal{J}}\right)$ in the category of chain complexes in general. Therefore, we define a universal enriched category $\operatorname{TwSum}\left(P_{\mathcal{J}}\right)$ generated by the formal image of the tensor products $w\left(x_{j}, j \in \mathcal{J}\right) \in$ $\operatorname{ob}\left(\operatorname{cat}\left(P_{\mathcal{J}}\right)\right)$ under such direct sum, suspension and twisting operations. If we put all these operations together, then we get the notion of a twisted direct sum which we formalize in our definition. Let us simply mention that we use formal tensor products $\mathbb{K} e \otimes V$, where $\mathbb{K} e$ is the free $\mathbb{K}$-module spanned by a homogeneous element of degree $d=\operatorname{deg}(e)$, to create a $d$-fold suspension operation $\Sigma^{d}: C \mapsto \Sigma^{d} C$. In the sequel, our idea is to define universal models of the homotopical construction which we need to work out our problems in this enriched category $\operatorname{TwSum}\left(P_{\mathcal{J}}\right)$.

2.2.1 Construction of the category of universal twisted sums Let $\mathcal{J}$ be a small category and $P_{\mathcal{J}}$ the associated $\mathrm{ob}(\mathcal{J})$-colored dg prop. Our goal is to build from $\operatorname{cat}\left(P_{\mathcal{J}}\right)$ a certain dg monoidal category $\operatorname{Tw} \operatorname{Sum}\left(P_{\mathcal{J}}\right)$ called its category of universal twisted sums. The objects are pairs

$$
\left(\bigoplus_{\underline{\alpha} \in A}\left(\mathbb{K}_{\underline{\alpha}}\right) \otimes\left(x_{\alpha_{1}} \otimes \cdots \otimes x_{\alpha_{n}}\right), \mathrm{tw}\right),
$$

where

- the first term $\bigoplus_{\underline{\alpha} \in A}$ is a formal sum over a finite set $A$ of multi-indices $\alpha=$ $\left(\alpha_{1}, \ldots, \alpha_{n}\right)$ of formal tensor products $\left(\mathbb{K} \mathbb{e}_{\alpha}\right) \otimes\left(x_{\alpha_{1}} \otimes \cdots \otimes x_{\alpha_{n}}\right)$, where $x_{\alpha_{1}} \otimes$ $\cdots \otimes x_{\alpha_{n}}$ is an object of cat $\left(P_{\mathcal{J}}\right)$ and we consider the graded $\mathbb{K}$-module $\mathbb{K}_{\underline{\alpha}}$ generated by a homogeneous element $\mathbb{e}_{\underline{\alpha}}$ of a certain degree $d_{\underline{\alpha}}=\operatorname{deg}\left(\mathbb{e}_{\underline{\alpha}}\right)$;

- the second term represents a collection of homomorphisms

$$
\mathrm{tw}_{\underline{\alpha} \underline{\beta}} \in \mathbb{e}_{\underline{\alpha}} \otimes \mathbb{e}_{\underline{\beta}}^{\vee} \otimes \operatorname{Hom}_{\mathrm{cat}\left(P_{\mathcal{J}}\right)}\left(x_{\beta_{1}} \otimes \cdots \otimes x_{\beta_{m}}, x_{\alpha_{1}} \otimes \cdots \otimes x_{\alpha_{n}}\right),
$$

indexed by the couples $(\underline{\alpha}, \beta) \in A^{2}$, homogeneous of degree -1 , that satisfy the relation of twisting cochains

$$
\delta\left(\mathrm{tw}_{\underline{\alpha} \underline{\beta}}\right)+\sum_{\underline{\gamma} \in A} \mathrm{tw} \underline{w}_{\underline{\alpha}} \circ \mathrm{tw}_{\underline{\gamma} \underline{\beta}}=0
$$

in the dg-module

$$
\mathbb{e}_{\underline{\alpha}} \otimes \mathbb{e}_{\underline{\beta}}^{\vee} \otimes \operatorname{Hom}_{\operatorname{cat}\left(P_{\mathcal{J}}\right)}\left(x_{\beta_{1}} \otimes \cdots \otimes x_{\beta_{m}}, x_{\alpha_{1}} \otimes \cdots \otimes x_{\alpha_{n}}\right),
$$

for every couple $(\underline{\alpha}, \underline{\beta}) \in A^{2}$ of sequences of colors. The notation $\mathbb{e}_{\beta}^{\vee}$ represents an element which is dual to $\mathbb{e}_{\underline{\beta}}$, homogeneous of degree $\operatorname{deg}\left(\mathbb{e}_{\beta}^{\vee}\right) \stackrel{-}{=}-\operatorname{deg}\left(\mathbb{e}_{\underline{\beta}}\right)$, and we use the relation $\mathbb{e}_{\underline{\beta}}^{\vee}\left(\mathbb{e}_{\underline{\beta}}\right)=1$ when we form the composites $\operatorname{tw}_{\underline{\alpha}} \underline{\underline{\gamma}} \circ \operatorname{tw}_{\underline{\gamma}} \underline{\beta}$. 
We define the dg-modules of homomorphisms of $\operatorname{TwSum}\left(P_{\mathcal{J}}\right)$ as the twisted sums of dg-modules; that is, for

$$
\begin{aligned}
& L=\left(\bigoplus_{\underline{\beta} \in B}\left(\mathbb{K}_{\underline{\beta}}\right) \otimes\left(x_{\beta_{1}} \otimes \cdots \otimes x_{\beta_{m}}\right), \mathrm{tw}_{L}\right), \\
& K=\left(\bigoplus_{\underline{\alpha} \in A}\left(\mathbb{K}_{\underline{\alpha}}\right) \otimes\left(x_{\alpha_{1}} \otimes \cdots \otimes x_{\alpha_{n}}\right), \mathrm{t} K\right),
\end{aligned}
$$

we define

$\operatorname{Hom}_{\mathrm{TwSum}\left(P_{\mathcal{J}}\right)}(L, K):=$

$$
\left(\bigoplus_{(\underline{\beta} \underline{\alpha}) \in B \times A} \mathbb{K} e_{\underline{\alpha}} \otimes \mathbb{K}_{\underline{\underline{\beta}}}^{\vee} \otimes \operatorname{Hom}_{\mathrm{cat}\left(P_{\mathcal{J}}\right)}\left(x_{\beta_{1}} \otimes \cdots \otimes x_{\beta_{m}}, x_{\alpha_{1}} \otimes \cdots \otimes x_{\alpha_{n}}\right), \partial\right),
$$

with twisting homomorphism $\partial:\left(f_{\beta} \underline{\alpha}\right) \mapsto\left(\partial(f)_{\beta} \underline{\alpha}\right)$ such that

$$
\partial(f)_{\underline{\beta} \underline{\alpha}}=\sum_{\underline{\gamma} \in B} \operatorname{tw}_{\underline{\beta}} \underline{\gamma} \circ f_{\underline{\gamma} \underline{\alpha}}-\sum_{\underline{\gamma} \in A} \operatorname{sign}(f) f_{\underline{\beta} \underline{\gamma}} \circ \operatorname{tw}_{\underline{\gamma}} \underline{\alpha}
$$

for every couple $(\underline{\alpha}, \underline{\beta})$ of sequences of colors, where $\operatorname{sign}(f)$ is a sign depending on $f$.

Claim This endows $\operatorname{Tw} \operatorname{Sum}\left(P_{\mathcal{J}}\right)$ with a dg category structure.

Proof We equip this dg hom $\operatorname{Hom}_{\mathrm{TwSum}\left(\boldsymbol{P}_{\mathcal{J}}\right)}(K, L)$ with the total differential $\delta+\partial$, where $\delta$ is the internal differential induced by the differential of $P$ and $\partial$ is the twisting homomorphism. The fact that $(\delta+\partial)^{2}=0$ follows from the relation of twisting cochains satisfied by the tw with respect to $\delta$. Indeed, for each $\underline{\beta} \in B, \underline{\alpha} \in A$, we have

$$
(\delta+\partial)^{2}(f)_{\underline{\beta}, \underline{\alpha}}=\left(\delta(\partial)+\partial^{2}\right)(f)_{\underline{\beta}, \underline{\alpha}},
$$

where $\delta(\partial)$ is the usual differential of a homomorphism defined by the commutator

$$
\delta(\partial)=\delta \circ \partial-(-1)^{\operatorname{deg}(\partial)} \partial \circ \delta=\delta \circ \partial+\partial \circ \delta .
$$

We have

$$
\delta(\partial)(f)_{\underline{\beta}, \underline{\alpha}}=\delta\left(\partial(f)_{\underline{\beta}, \underline{\alpha}}\right)+\partial(\delta(f))_{\underline{\beta}, \underline{\alpha}}=\delta\left(\mathrm{tw}_{\underline{\beta}, \underline{\alpha}}\right)(f),
$$


and

$$
\begin{aligned}
\partial^{2}(f)_{\underline{\beta}, \underline{\alpha}}=\partial(\partial(f))_{\underline{\beta}, \underline{\alpha}} & =\sum_{\underline{\gamma} \in B} \mathrm{tw}_{\underline{\beta} \underline{\gamma}} \circ \partial(f)_{\underline{\gamma} \underline{\alpha}}-\sum_{\underline{\gamma} \in A} \operatorname{sign}(\partial(f)) \partial(f)_{\underline{\beta} \underline{\underline{\gamma}}} \circ \mathrm{tw}_{\underline{\gamma} \underline{\alpha}} \\
& =\sum_{\underline{\gamma} \in B} \mathrm{tw}_{\underline{\beta} \underline{\gamma}} \circ \partial(f)_{\underline{\gamma} \underline{\alpha}}+\sum_{\underline{\gamma} \in A} \operatorname{sign}(f) \partial(f)_{\underline{\beta} \underline{\underline{\gamma}}} \circ \mathrm{tw}_{\underline{\gamma} \underline{\alpha}} \\
& =\left(\sum_{\underline{\gamma} \in B} \mathrm{tw}_{\underline{\beta} \underline{\gamma}} \circ \operatorname{tw}_{\underline{\gamma} \underline{\alpha}}\right)(f)
\end{aligned}
$$

because $\operatorname{sign}(\partial(f))=\operatorname{sign}(f)-1$ (the homomorphism $\partial$ is of degree -1$)$, so

$$
(\delta+\partial)^{2}(f)_{\underline{\beta}, \underline{\alpha}}=\left(\delta\left(\mathrm{tw}_{\underline{\beta}, \underline{\alpha}}\right)+\sum_{\underline{\gamma} \in B} \mathrm{tw}_{\underline{\beta}} \underline{\underline{\gamma}} \circ \mathrm{tw}_{\underline{\gamma} \underline{\alpha}}\right)(f)=0 .
$$

For each object

$$
K=\left(\bigoplus_{\underline{\alpha}} \mathbb{K}_{\underline{\alpha}} \otimes\left(x_{\alpha_{1}} \otimes \cdots \otimes x_{\alpha_{n}}\right), \mathrm{tw}_{K}\right)
$$

of $\operatorname{TwSum}\left(P_{\mathcal{J}}\right)$, the associated identity element in the $\operatorname{dg} \operatorname{hom} \operatorname{Hom}_{\mathrm{TwSum}\left(P_{\mathcal{J}}\right)}(K, K)$ is the 0 -cycle defined by

$$
\bigoplus_{\underline{\alpha}}\left(\mathbb{K e}_{\underline{\alpha}}\right) \otimes \mathbb{K}_{\underline{\alpha}}^{\vee} \otimes \operatorname{id}_{x_{\alpha_{1}} \otimes \cdots \otimes x_{\alpha_{n}}},
$$

where $\operatorname{id}_{x_{\alpha_{1}} \otimes \cdots \otimes x_{\alpha_{n}}}$ is the identity on the object $x_{\alpha_{1}} \otimes \cdots \otimes x_{\alpha_{n}}$ of $\operatorname{cat}\left(P_{\mathcal{J}}\right)$. The composition law

$$
\operatorname{Hom}_{\mathrm{TwSum}\left(P_{\mathcal{J}}\right)}(K, L) \otimes \operatorname{Hom}_{\mathrm{TwSum}\left(P_{\mathcal{J}}\right)}(L, M) \rightarrow \operatorname{Hom}_{\mathrm{TwSum}\left(P_{\mathcal{J}}\right)}(K, M)
$$

on such dg homs is then defined by the composition of $\operatorname{dg} \operatorname{homs}$ in $\operatorname{cat}\left(P_{\mathcal{J}}\right)$ and the relation $\mathbb{e}_{\underline{\alpha}}^{\vee}\left(\mathbb{e}_{\underline{\alpha}}\right)=1$ on matching colors. The compatibility of this composition with the twisted differentials of the dg homs is automatic.

2.2.2 The tensor structure on a category of universal twisted sums The category $\operatorname{TwSum}\left(P_{\mathcal{J}}\right)$ is equipped with a dg enriched symmetric monoidal structure, defined by the natural distribution formula at the level of objects; that is, for

$$
\begin{aligned}
& K=\left(\bigoplus_{\underline{\alpha}}\left(\mathbb{K}_{\underline{\alpha}}\right) \otimes\left(x_{\alpha_{1}} \otimes \cdots \otimes x_{\alpha_{m}}\right), \mathrm{tw}_{K}\right), \\
& L=\left(\bigoplus_{\underline{\beta}}\left(\mathbb{K}_{\underline{\beta}}\right) \otimes\left(x_{\beta_{1}} \otimes \cdots \otimes x_{\beta_{n}}\right), \mathrm{tw}_{L}\right),
\end{aligned}
$$


we define

$K \otimes L:=\left(\bigoplus_{\underline{\alpha}, \underline{\beta}}\left(\mathbb{K}_{\underline{\alpha}} \otimes \mathbb{e}_{\underline{\beta}}\right) \otimes\left(x_{\alpha_{1}} \otimes \cdots \otimes x_{\alpha_{m}} \otimes x_{\beta_{1}} \otimes \cdots \otimes x_{\beta_{n}}\right), \mathrm{tw}_{K} \otimes \mathrm{id}+\mathrm{id} \otimes \mathrm{tw}_{L}\right)$, where we use the horizontal compositions

$\left(\mathbb{K}_{\underline{\gamma}} \otimes \mathbb{K}_{\underline{\alpha}}^{\vee} \otimes \operatorname{Hom}_{\text {cat }\left(P_{\mathcal{J}}\right)}\left(x_{\alpha_{1}} \otimes \cdots \otimes x_{\alpha_{m}}, x_{\gamma_{1}} \otimes \cdots \otimes x_{\gamma_{p}}\right)\right)$

$\otimes\left(\mathbb{K}_{\underline{\underline{\delta}}} \otimes \mathbb{K}_{\underline{\underline{\beta}}}^{\vee} \otimes \operatorname{Hom}_{\mathrm{cat}\left(\boldsymbol{P}_{\mathcal{J}}\right)}\left(x_{\beta_{1}} \otimes \cdots \otimes x_{\beta_{n}}, x_{\delta_{1}} \otimes \cdots \otimes x_{\delta_{q}}\right)\right)$

$\stackrel{\otimes}{\rightarrow}\left(\mathbb{K}_{e_{\underline{\gamma}}} \otimes \mathbb{K}_{\underline{\underline{\delta}}}\right) \otimes\left(\mathbb{K}_{\underline{\underline{\alpha}}} \otimes \mathbb{K}_{\underline{\beta}}\right)^{\vee} \otimes \operatorname{Hom}_{\mathrm{cat}\left(\boldsymbol{P}_{\mathcal{J}}\right)}\left(x_{\alpha_{1}} \otimes \cdots \otimes x_{\alpha_{m}} \otimes x_{\beta_{1}} \otimes \cdots \otimes x_{\beta_{n}}\right.$, $\left.x_{\gamma_{1}} \otimes \cdots \otimes x_{\gamma_{p}} \otimes x_{\delta_{1}} \otimes \cdots \otimes x_{\delta_{q}}\right)$

to define the formal twisted cochain ${ }^{w_{W}}{ }_{K} \otimes \mathrm{id}+\mathrm{id} \otimes \mathrm{tw}_{L}$ of this object $K \otimes L$. An analogous construction holds at the level of homomorphisms.

Each object $x_{\alpha_{1}} \otimes \cdots \otimes x_{\alpha_{n}} \in \operatorname{cat}\left(P_{\mathcal{J}}\right)$ is naturally identified with the trivial twisted sum $K=\left(\mathbb{K}_{0} \otimes\left(x_{\alpha_{1}} \otimes \cdots \otimes x_{\alpha_{n}}\right), 0\right)$ in $\operatorname{Tw} \operatorname{Sum}\left(P_{\mathcal{J}}\right)$, where $\operatorname{deg}\left(\mathbb{e}_{0}\right)=0 \Rightarrow \mathbb{K} \mathbb{e}_{0}=\mathbb{K}$. In particular, to each $x_{\alpha_{i}}$ corresponds a trivial twisted sum $K_{\alpha_{i}}=\left(\mathbb{K e}_{0} \otimes x_{\alpha_{i}}, 0\right)$. This defines a functor

$$
\operatorname{cat}\left(P_{\mathcal{J}}\right) \rightarrow \operatorname{TwSum}\left(P_{\mathcal{J}}\right)
$$

The category of universal twisted sums satisfies the following universal property with respect to this functor.

Lemma 2.6 For every symmetric monoidal dg functor $R$ : $\operatorname{cat}\left(P_{\mathcal{J}}\right) \rightarrow \mathcal{C}$ (that is, every $P_{\mathcal{J}}$-algebra), there exists a canonical factorization:

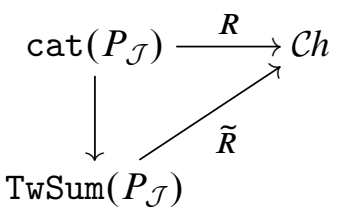

Proof We construct $\widetilde{R}$ by first setting $\widetilde{R}\left(K_{\alpha_{i}}\right)=R\left(x_{\alpha_{i}}\right)$ so that the diagram commutes. Then, for any object

$$
\left(\bigoplus_{\underline{\alpha}}\left(\mathbb{K}_{\underline{\alpha}}\right) \otimes\left(x_{\alpha_{1}} \otimes \cdots \otimes x_{\alpha_{n}}\right), \mathrm{tw}\right)
$$


of $\operatorname{TwSum}\left(P_{\mathcal{J}}\right)$, we define

$$
\begin{aligned}
\tilde{R}\left(\bigoplus_{\underline{\alpha}}\left(\mathbb{K}_{\underline{\alpha}}\right) \otimes\left(x_{\alpha_{1}} \otimes \cdots \otimes x_{\alpha_{n}}\right), \mathrm{tw}\right) \\
=\left(\bigoplus_{\underline{\alpha}}\left(\mathbb{K}_{\underline{\underline{\alpha}}}\right) \otimes\left(R\left(x_{\alpha_{1}}\right) \otimes \cdots \otimes R\left(x_{\alpha_{n}}\right)\right), R(\mathrm{tw})\right),
\end{aligned}
$$

where the left-hand term is built with the direct sum and tensor product of $\mathcal{C}$. The differential of $\widetilde{R}\left(\bigoplus_{\underline{\alpha}}\left(\mathbb{K}_{\underline{\alpha}}\right) \otimes\left(x_{\alpha_{1}} \otimes \cdots \otimes x_{\alpha_{n}}\right)\right.$, tw $)$ is then defined on each component of this direct sum by the sum of the differential of $R\left(x_{\alpha_{1}}\right) \otimes \cdots \otimes R\left(x_{\alpha_{n}}\right)$ with a twisting cochain $R(\mathrm{tw})$ defined as follows. Since $R$ is a symmetric monoidal $\mathrm{dg}$ functor, it induces a morphism of chain complexes

$$
\begin{aligned}
R_{x_{\beta_{1}} \otimes \cdots \otimes x_{\beta_{m}}, x_{\alpha_{1}} \otimes \cdots \otimes x_{\alpha_{n}}}: \operatorname{Hom}_{\mathrm{cat}\left(P_{\mathcal{J}}\right)}\left(x_{\beta_{1}} \otimes \cdots \otimes x_{\beta_{m}}, x_{\alpha_{1}} \otimes \cdots \otimes x_{\alpha_{n}}\right) \\
\rightarrow \operatorname{Hom}_{C h}\left(R\left(x_{\beta_{1}}\right) \otimes \cdots \otimes R\left(x_{\beta_{m}}\right), R\left(x_{\alpha_{1}}\right) \otimes \cdots \otimes R\left(x_{\alpha_{n}}\right)\right),
\end{aligned}
$$

so that the collection $R(\mathrm{tw})=\left\{R\left(\mathrm{t}_{\mathrm{w}} \underline{\alpha} \underline{\beta}\right)\right\}_{\underline{\alpha}} \underline{\beta}$ is well defined by

$$
\begin{aligned}
R\left(\mathrm{tw}_{\underline{\alpha}} \underline{\beta}\right) & =R_{x_{\beta_{1}} \otimes \cdots \otimes x_{\beta m}, x_{\alpha_{1}} \otimes \cdots \otimes x_{\alpha_{n}}}\left(\mathrm{tw}_{\underline{\alpha}} \underline{\beta}\right) \\
& \in \mathbb{e}_{\underline{\alpha}} \otimes \mathbb{e}_{\underline{\beta}}^{\vee} \otimes \operatorname{Hom}_{C h}\left(R\left(x_{\beta_{1}}\right) \otimes \cdots \otimes R\left(x_{\beta_{m}}\right), R\left(x_{\alpha_{1}}\right) \otimes \cdots \otimes R\left(x_{\alpha_{n}}\right)\right) .
\end{aligned}
$$

This collection satisfies the relation of twisting cochains because $R$ is a symmetric monoidal dg functor and the collection $\{\mathrm{t} \underline{\alpha} \underline{\beta}\}_{\underline{\alpha}} \underline{\beta}$ satisfies the relation of twisting cochains in $\operatorname{TwSum}\left(P_{\mathcal{J}}\right)$.

2.2.3 Functorial diagrams of algebras Our purpose is to use categories of universal twisted sums to construct diagrams of dg $P$-algebras "functorial in their variables" in a suitable sense.

Recall that the colored dg prop $P_{\mathcal{J}}$ parametrizing $\mathcal{J}$-diagrams of $P$-algebras is equivalent to the datum of a symmetric monoidal dg category $\operatorname{cat}\left(P_{\mathcal{J}}\right)$. Algebras over $P_{\mathcal{J}}$ are then strict symmetric monoidal dg functors cat $\left(P_{\mathcal{J}}\right) \rightarrow \mathcal{C h}$, and morphisms of $P_{\mathcal{J}}$-algebras are natural transformations preserving the strict symmetric monoidal dg structures. Such a natural transformation corresponds to a natural transformation of $\mathcal{J}$-diagrams of $P$-algebras.

Now let $A, B: \operatorname{cat}\left(P_{\mathcal{J}}\right) \rightarrow \mathcal{C} h$ be two such algebras, and $\phi: A \Rightarrow B$ be a strict symmetric monoidal dg natural transformation. Recall that, according to Lemma 2.6, such functors lift to strict symmetric monoidal dg functors $\tilde{A}, \widetilde{B}: \operatorname{Tw} \operatorname{Sum}\left(P_{\mathcal{J}}\right) \rightarrow \mathcal{C}$. We want to prove that such a lift works similarly for symmetric monoidal dg natural transformations between such functors. 
Lemma 2.7 The natural transformation $\phi$ lifts to a strict symmetric monoidal $d g$ natural transformation $\widetilde{\phi}: \widetilde{A} \Rightarrow \widetilde{B}$.

Proof To see this, let us first recall from [15] the notion of enriched natural transformation in the case where the categories are enriched over $\mathcal{C} h$. Let $F, G: \mathcal{C} \rightarrow \mathcal{D}$ be two $\mathrm{dg}$ functors and $\operatorname{Hom}_{\mathcal{C}}(-,-), \operatorname{Hom}_{\mathcal{D}}(-,-)$ be respectively the $\mathrm{dg}$ homs of $\mathcal{C}$ and $\mathcal{D}$. A dg natural transformation $\tau: F \Rightarrow G$ is a collection of chain morphisms

$$
\left\{\tau(x): \mathbb{K} \rightarrow \operatorname{Hom}_{\mathcal{D}}(F(x), G(x))\right\}_{x \in \mathrm{ob}(\mathcal{C})},
$$

that is, of 0 -cycles in the complexes $\operatorname{Hom}_{\mathcal{D}}(F(x), G(x))$ indexed by the objects $x$ of $\mathcal{C}$. For every $x, y \in \operatorname{ob}(\mathcal{C})$, this collection makes the following diagram commutative:

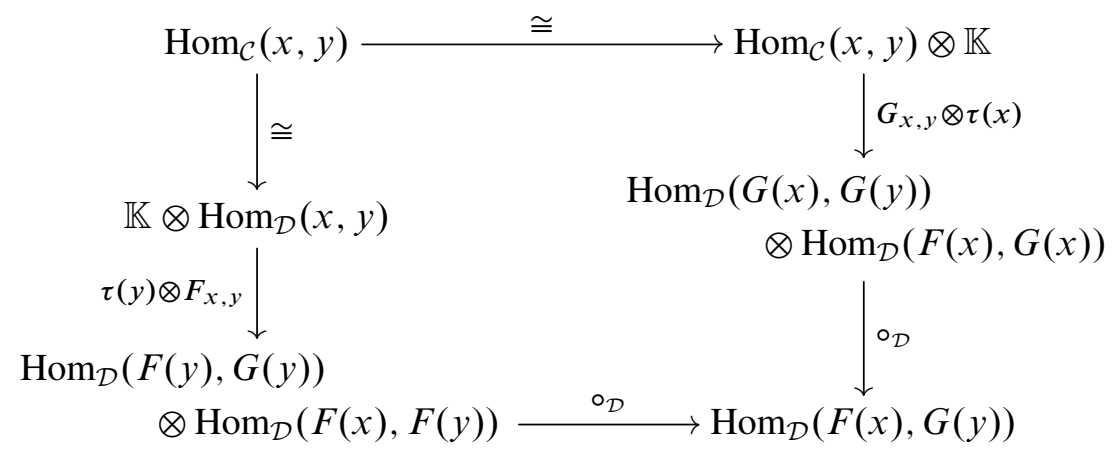

For any object

$$
K=\left(\bigoplus_{\underline{\alpha}}\left(\mathbb{K}_{\underline{\alpha}}\right) \otimes\left(x_{\alpha_{1}} \otimes \cdots \otimes x_{\alpha_{n}}\right), \text { tw }_{K}\right)
$$

of $\operatorname{Tw} \operatorname{Sum}\left(P_{\mathcal{J}}\right)$, we define the associated 0 -cycle $\widetilde{\phi}$ in

$\operatorname{Hom}_{C h}\left(\left(\bigoplus_{\underline{\alpha}}\left(\mathbb{K}_{\underline{\alpha}}\right) \otimes\left(A\left(x_{\alpha_{1}}\right) \otimes \cdots \otimes A\left(x_{\alpha_{n}}\right)\right), A\left(\mathrm{t}_{w_{K}}\right)\right)\right.$,

$$
\left.\left(\bigoplus_{\underline{\alpha}}\left(\mathbb{K}_{\underline{\alpha}}\right) \otimes\left(B\left(x_{\alpha_{1}}\right) \otimes \cdots \otimes B\left(x_{\alpha_{n}}\right)\right), B\left(\mathrm{tw}_{K}\right)\right)\right)
$$

by

$$
\tilde{\phi}(K)=\bigoplus_{\underline{\alpha}}\left(\mathbb{K} e_{\underline{\alpha}}\right) \otimes\left(\phi\left(x_{\alpha_{1}}\right) \otimes \cdots \otimes \phi\left(x_{\alpha_{n}}\right)\right) .
$$

We have to prove that this form a 0 -cycle, thus that

$$
\left(\delta+B\left(\mathrm{tw}_{K}\right)\right) \circ \tilde{\phi}(K)=\tilde{\phi}(K) \circ\left(\delta+A\left(\mathrm{tw}_{K}\right)\right) .
$$

The equality

$$
\delta \circ \widetilde{\phi}(K)=\widetilde{\phi}(K) \circ \delta
$$


follows from the fact that each $\phi\left(x_{\alpha_{i}}\right): A\left(x_{\alpha_{i}}\right) \rightarrow B\left(x_{\alpha_{i}}\right)$ is a morphism of chain complexes and the differential $\delta$ is the differential of $B\left(x_{\alpha_{1}}\right) \otimes \cdots \otimes B\left(x_{\alpha_{n}}\right)$ on the left-hand side of the equality and of $A\left(x_{\alpha_{1}}\right) \otimes \cdots \otimes A\left(x_{\alpha_{n}}\right)$ on the right-hand side. The equality

$$
B\left(\mathrm{tw}_{K}\right) \circ \tilde{\phi}(K)=\tilde{\phi}(K) \circ A\left(\mathrm{tw}_{K}\right)
$$

follows from the definition of $A\left(\mathrm{tw}_{K}\right)$ as

$$
A\left(\mathrm{tw}_{K}\right)=\left\{A_{x_{\beta_{1}} \otimes \cdots \otimes x_{\beta m}, x_{\alpha_{1}} \otimes \cdots \otimes x_{\alpha_{n}}}\left(\left(\mathrm{tw}_{K}\right)_{\underline{\alpha}} \underline{\beta}\right)\right\}_{\underline{\alpha}} \underline{\beta}
$$

(and the same for $B\left(\mathrm{tw}_{K}\right)$ ), the fact that $\phi$ is a dg natural transformation between $A$ and $B$, and the definition of $\tilde{\phi}(K)$ in terms of the $\phi\left(x_{\alpha_{i}}\right)$.

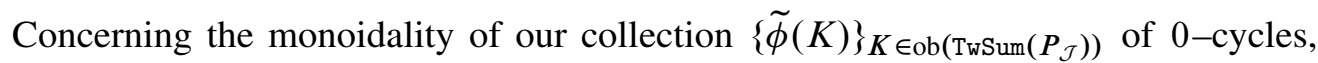
recall from Section 2.2.2 that the tensor product of two objects of $\operatorname{Tw} \operatorname{Sum}\left(P_{\mathcal{J}}\right)$ is defined by

$$
\underbrace{\left(\bigoplus_{\underline{\alpha}}\left(\mathbb{K e}_{\underline{\alpha}}\right) \otimes\left(x_{\alpha_{1}} \otimes \cdots \otimes x_{\alpha_{m}}\right), \mathrm{tw}_{K}\right)}_{K} \otimes \underbrace{\left(\bigoplus_{\underline{\beta}}\left(\mathbb{K e}_{\underline{\beta}}\right) \otimes\left(x_{\beta_{1}} \otimes \cdots \otimes x_{\beta_{n}}\right), \mathrm{t}{ }_{L}\right)}_{L}
$$

$:=\left(\bigoplus_{\underline{\alpha}, \underline{\beta}}\left(\mathbb{K}_{\underline{\alpha}} \otimes \mathbb{e}_{\underline{\beta}}\right) \otimes\left(x_{\alpha_{1}} \otimes \cdots \otimes x_{\alpha_{m}} \otimes x_{\beta_{1}} \otimes \cdots \otimes x_{\beta_{n}}\right), \mathrm{tw}_{K} \otimes \mathrm{id}+\mathrm{id} \otimes \mathrm{tw}_{L}\right)$

$$
=K \otimes L
$$

and that the functors $\tilde{A}, \widetilde{B}: \operatorname{TwSum}\left(P_{\mathcal{J}}\right) \rightarrow \mathcal{C} h$ associated to $A, B: \operatorname{cat}\left(P_{\mathcal{J}}\right) \rightarrow \mathcal{C} h$ are defined by

$$
\begin{aligned}
\tilde{A}\left(\bigoplus_{\underline{\alpha}}\left(\mathbb{K}_{\underline{\alpha}}\right) \otimes\left(x_{\alpha_{1}} \otimes \cdots \otimes x_{\alpha_{n}}\right), \mathrm{tw}\right) \\
=\left(\bigoplus_{\underline{\alpha}}\left(\mathbb{K}_{\underline{\alpha}}\right) \otimes\left(A\left(x_{\alpha_{1}}\right) \otimes \cdots \otimes A\left(x_{\alpha_{n}}\right)\right), A\left(\mathrm{t}_{\mathrm{w}}\right)\right) .
\end{aligned}
$$

We have natural isomorphisms

$$
a_{K \otimes L}: \widetilde{A}(K \otimes L) \stackrel{\cong}{\rightrightarrows} \widetilde{A}(K) \otimes \widetilde{A}(L), \quad b_{K} \otimes L: \widetilde{B}(K \otimes L) \stackrel{\cong}{\rightrightarrows} \widetilde{B}(K) \otimes \widetilde{B}(L),
$$

induced by natural isomorphisms

$$
A(\cdot \otimes \cdot) \stackrel{\cong}{\rightrightarrows} A(\cdot) \otimes A(\cdot), \quad B(\cdot \otimes \cdot) \stackrel{\cong}{\rightarrow} B(\cdot) \otimes B(\cdot),
$$


since $A$ and $B$ are symmetric monoidal functors. We have to check the commutativity of the square below:

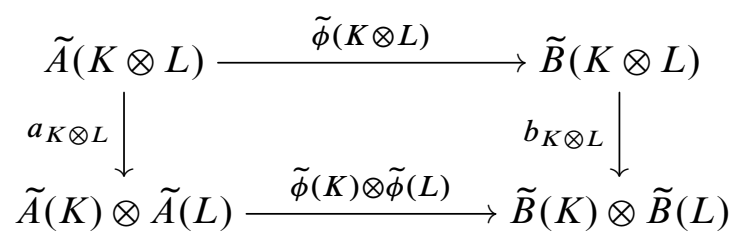

By construction of $\widetilde{A}, \widetilde{B}$ and $\widetilde{\phi}$, which are defined by applying $A, B$ and $\phi$ to each variable of the tensor powers defining the objects of $\operatorname{Tw} \operatorname{Sum}\left(P_{\mathcal{J}}\right)$, this boils down to the commutativity of such a monoidality square for $A, B$ and $\phi$, which holds because $\phi$ is a monoidal natural transformation.

The naturality of $\{\tilde{\phi}(K)\}_{K \in \mathrm{ob}\left(\operatorname{TwSum}\left(P_{\mathcal{J}}\right)\right)}$ follows directly from the naturality of $\phi$.

We consequently get two functors

$$
\widetilde{A}_{*}, \widetilde{B}_{*}: \operatorname{Tw} \operatorname{Sum}\left(P_{\mathcal{J}}\right)^{P} \rightarrow \mathcal{C} h^{P},
$$

that carry any $P$-algebra in $\operatorname{TwSum}\left(P_{\mathcal{J}}\right)$, represented by a symmetric monoidal functor $\widetilde{C}: \operatorname{cat}(P) \rightarrow \operatorname{TwSum}\left(P_{\mathcal{J}}\right)$, to the $P$-algebra in $\mathcal{C}$ represented by the composite functors $\widetilde{A} \widetilde{C}, \widetilde{B} \widetilde{C}: \operatorname{cat}(P) \rightarrow \mathcal{C} h$. We also have a natural transformation $\widetilde{\phi}_{*}: \widetilde{A}_{*} \Rightarrow \widetilde{B}_{*}$ between these functors on $P$-algebras.

For any small category $\mathcal{I}$, we get strict symmetric monoidal dg functors

$$
\widetilde{A}_{*}, \widetilde{B}_{*}:\left(\operatorname{Tw} \operatorname{Sum}\left(P_{\mathcal{J}}\right)^{P}\right)^{\mathcal{I}} \rightarrow\left(\mathcal{C} h^{P}\right)^{\mathcal{I}}
$$

and a strict symmetric monoidal dg natural transformation $\widetilde{\phi}_{*}: \widetilde{A}_{*} \Rightarrow \widetilde{B}_{*}$. This transformation consists in a collection of natural transformations of $\mathcal{I}$-diagrams of $\mathrm{dg}$ $P$-algebras

$$
\widetilde{\phi}_{*}(Y): \widetilde{A}_{*}(Y) \Rightarrow \widetilde{B}_{*}(Y)
$$

for every $Y \in\left(\operatorname{TwSum}\left(P_{\mathcal{J}}\right)^{P}\right)^{\mathcal{I}}$.

Thus, whenever we have an $\mathcal{I}$-diagram of $P$-algebras in $\operatorname{Tw} \operatorname{Sum}\left(P_{\mathcal{J}}\right)$, say $Y$, we can associate an $\mathcal{I}$-diagram of dg $P$-algebras $\widetilde{A}_{*}(Y)$ to any $\mathcal{J}$-diagram of $\underset{\widetilde{\phi_{*}}}{\operatorname{dg}} P$-algebras $A$, and a natural transformation of $\mathcal{I}$-diagrams of dg $P$-algebras $\widetilde{\phi}_{*}(Y): \widetilde{A}_{*}(Y) \Rightarrow \widetilde{B}_{*}(Y)$ to any natural transformation of $\mathcal{J}$-diagrams of dg $P$-algebras $\phi: A \Rightarrow B$. This result is equivalent to the following statement.

Proposition 2.8 Given an $\mathcal{I}$-diagram $Y$ of $P$-algebras in $\operatorname{TwSum}\left(P_{\mathcal{J}}\right)$, the above construction determines a functor

$$
\left(\mathcal{C} h^{P}\right)^{\mathcal{J}} \rightarrow\left(C h^{P}\right)^{\mathcal{I}}
$$


The main example to which we want to apply this construction is the following. Let $f: X \rightarrow Y$ be a morphism of chain complexes. Then it admits a functorial factorization by an acyclic cofibration (ie acyclic injection) followed by a fibration (ie a surjection). This factorization is explicitly given by

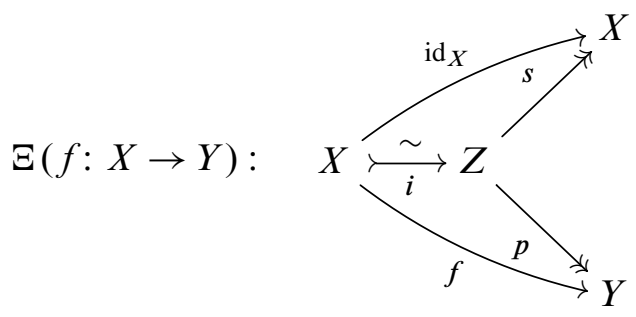

where

$$
Z=\left(\mathbb{K e}_{0} \otimes X \oplus \mathbb{K} e_{01} \otimes Y \oplus \mathbb{K} e_{1} \otimes Y, d_{Z}\right),
$$

with $\operatorname{deg}\left(\mathbb{e}_{0}\right)=\operatorname{deg}\left(\mathbb{e}_{1}\right)=0$ and $\operatorname{deg}\left(\mathbb{e}_{01}\right)=-1$. The differential $d_{Z}$ can be expressed in this direct sum by the matrix

$$
\left(\begin{array}{ccc}
d_{X} & 0 & 0 \\
f & -d_{Y} & -\mathrm{id} \\
0 & 0 & d_{Y}
\end{array}\right)=\left(\begin{array}{ccc}
d_{X} & 0 & 0 \\
0 & -d_{Y} & 0 \\
0 & 0 & d_{Y}
\end{array}\right)+\left(\begin{array}{ccc}
0 & 0 & 0 \\
f & 0 & -\mathrm{id} \\
0 & 0 & 0
\end{array}\right)
$$

where the first matrix of the sum is the differential of the direct sum

$$
\mathbb{K} e_{0} \otimes X \oplus \mathbb{K} e_{01} \otimes Y \oplus \mathbb{K} e_{1} \otimes Y
$$

and the second is a twisting $\mathrm{tw}_{Z}$, a map of degree -1 satisfying $\mathrm{tw}_{Z}^{2}=0$. The map $i$ sends $x \in X$ to $x \oplus 0 \oplus f(x)$ and $s$ and $p$ are respectively projections on the first and the third factor; that is, we have

$$
i=\left(\begin{array}{lll}
\text { id } & 0 & f
\end{array}\right), \quad s=\left(\begin{array}{c}
\mathrm{id} \\
0 \\
0
\end{array}\right) \quad \text { and } \quad p=\left(\begin{array}{c}
0 \\
0 \\
\mathrm{id}
\end{array}\right) .
$$

There is a diagram of chain complexes

$$
\Xi: \operatorname{Mor}(\mathcal{C h}) \rightarrow \operatorname{Fun}(\mathcal{Y}, \mathcal{C} h)
$$

functorial in its variables, where $\operatorname{Mor}(\mathrm{Ch})$ is the category whose objects are morphisms of chain complexes and morphisms are commutative squares, and $\mathcal{Y}$ is the small 
category whose objects and arrows are given by

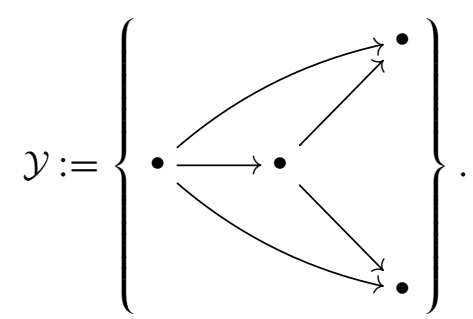

Our goal is to prove that for any cofibrant $\operatorname{dg}$ prop $P$, this functor induces a functor

$$
\Xi: \operatorname{Mor}\left(\mathcal{C} h^{P}\right) \rightarrow \operatorname{Fun}\left(\mathcal{Y}, \mathcal{C} h^{P}\right),
$$

that is, a functor

$$
\Xi:\left(\mathrm{Ch}^{P}\right)^{\bullet \rightarrow \bullet} \rightarrow\left(\mathcal{C} h^{P}\right)^{\mathcal{Y}}
$$

This means the following.

Theorem 2.9 Let $P$ be a cofibrant dg prop. The functorial factorization of morphisms of chain complexes described above lifts to a functorial factorization of $P$-algebra morphisms into an acyclic injection followed by a surjection.

Proof The general strategy is to prove that the diagram in $\operatorname{TwSum}\left(P_{x \rightarrow y}\right)$ associated to $\Xi(f: X \rightarrow Y)$ is actually a diagram in $\operatorname{TwSum}\left(P_{X \rightarrow y}\right)$, and then apply Proposition 2.8.

Let $f: X \rightarrow Y$ be a morphism of chain complexes and $P_{x \rightarrow y}$ the 2-colored dg prop of $P$-algebra morphisms. In this proof, we will use the short notation

$$
\mathrm{Tw}:=\operatorname{TwSum}\left(P_{x \rightarrow y}\right) .
$$

We can associate to the diagram of chain complexes $\Xi(f: X \rightarrow Y)$ a diagram $\Xi(f: x \rightarrow y)$ in $\mathrm{Tw}$ so that Proposition 2.8 applies. For this, recall that the colors $x$ and $y$ are embedded into Tw as the objects $\left(\mathbb{K}_{0} \otimes x, 0\right)$ and $\left(\mathbb{K}_{1} \otimes y, 0\right)$. We will denote by $f$ both the operation of $P_{x \rightarrow y}$ corresponding to $f$ and the morphism $\left(\mathbb{K e}_{0} \otimes x, 0\right) \rightarrow\left(\mathbb{K e}_{1} \otimes y, 0\right)$ in Tw. The object $z$ of Tw corresponding to $Z$ is defined to be

$$
\left(\mathbb{K} e_{0} \otimes x \oplus \mathbb{K} e_{01} \otimes y \oplus \mathbb{K} e_{1} \otimes y, t_{w_{z}}\right),
$$

with

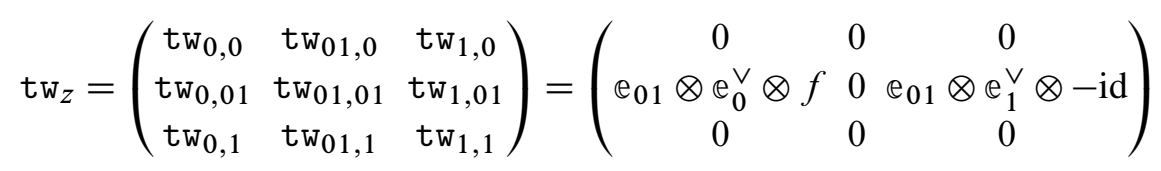


representing the twisting part tw $Z$ of $Z$. The maps $i$ and $p$ of $\Xi(f: x \rightarrow y)$ are then defined similarly to those of $\Xi(f: X \rightarrow Y)$.

The endomorphism dg prop $\operatorname{End}_{(\Xi(f: x \rightarrow y), T w)}$ projects to the endomorphism dg prop $\operatorname{End}_{\left(f, \mathrm{Tw}_{\mathrm{w}}\right)}$ of the subdiagram $f: x \rightarrow y$, hence we have a fibration of dg props

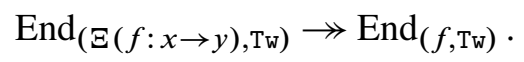

We will denote these dg props by $\operatorname{End}_{\Xi(f: x \rightarrow y)}$ and $\operatorname{End}_{f}$ for short. We have to prove that this fibration is acyclic. For this, we consider the following commutative diagram of $\mathbb{S}$-biobjects:

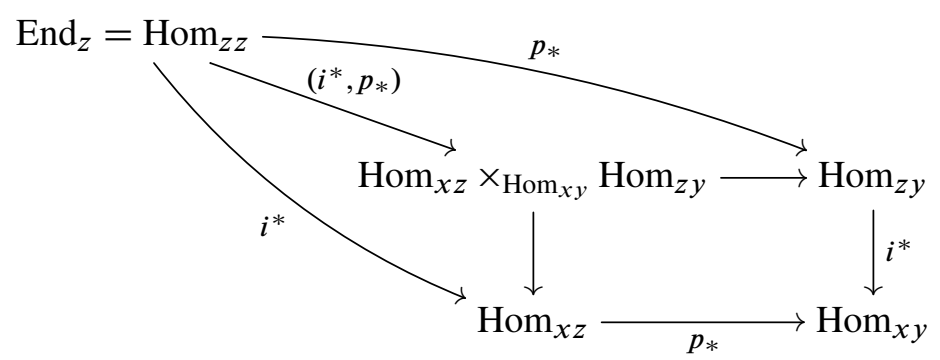

where $\operatorname{Hom}_{z z}(m, n)=\operatorname{Hom}_{\mathrm{Tw}}\left(z^{\otimes m}, z^{\otimes n}\right)$. Limits of $\mathbb{S}$-biobjects are created pointwise, so for every $(m, n) \in \mathbb{N}^{2}$ we have a commutative diagram as follows:

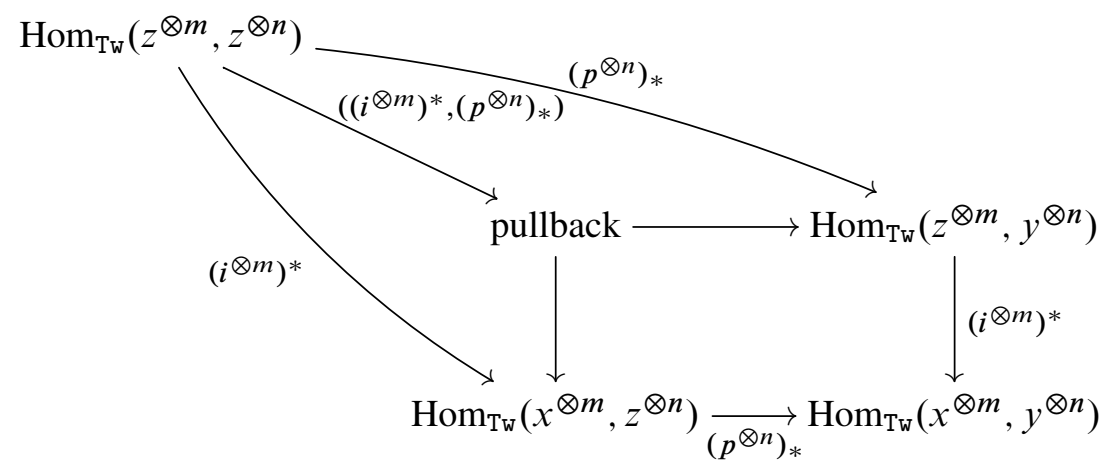

We have to check that $\left(\left(i^{\otimes m}\right)^{*},\left(p^{\otimes n}\right)_{*}\right)$ is an acyclic fibration. Since acyclic fibrations of $\mathbb{S}$-biobjects are determined pointwise, we deduce that

$$
\left(i^{*}, p_{*}\right): \operatorname{End}_{z} \stackrel{\sim}{\rightarrow} \operatorname{Hom}_{x z} \times \operatorname{Hom}_{x y} \operatorname{Hom}_{y z}
$$

is an acyclic fibration of $\Sigma$-objects. Let us consider now the base extensions

$$
\operatorname{End}_{x} \times_{\operatorname{Hom}_{x z}} \operatorname{End}_{z} \times_{\mathrm{Hom}_{z}} \operatorname{End}_{y}=\operatorname{End}_{\Xi(f: x \rightarrow y)},
$$

$\operatorname{End}_{x} \times_{\operatorname{Hom}_{x z}}\left(\operatorname{Hom}_{x z} \times_{\operatorname{Hom}_{x y}} \operatorname{Hom}_{z y}\right) \times_{\operatorname{Hom}_{z y}} \operatorname{End}_{y}=\operatorname{End}_{f}$. 
Acyclic fibrations are stable under base extensions, and acyclic fibrations of dg props are determined in the category of $\mathbb{S}$-biobjects under the forgetful functor, so we finally get the desired acyclic fibration of dg props

$$
\operatorname{End}_{x} \times_{\operatorname{Hom}_{x z}}\left(i^{*}, p_{*}\right) \times_{\operatorname{Hom}_{z y}} \operatorname{End}_{y}: \operatorname{End}_{\Xi(f: x \rightarrow y)} \stackrel{\sim}{\rightarrow} \operatorname{End}_{f} .
$$

Now let us denote $X_{b}=\mathbb{K e}_{0}, Y_{b}=\mathbb{K}_{1}$ and $f_{b}: X_{b} \rightarrow Y_{b}$ the morphism sending $\mathbb{e}_{0}$ to $\mathbb{e}_{1}$. This morphism admits a factorization

$$
X_{b} \underset{i_{b}}{\stackrel{\sim}{\longrightarrow}} Z_{b} \underset{p_{b}}{\longrightarrow} Y_{b}
$$

Our goal is to prove that for all natural integers $m$ and $n$, we have isomorphisms of chain complexes

$$
\begin{aligned}
& \operatorname{Hom}_{\mathrm{Tw}}\left(z^{\otimes m}, z^{\otimes n}\right) \cong \operatorname{Hom}_{C h}\left(Z_{b}^{\otimes m}, Z_{b}^{\otimes n}\right) \otimes P(m, n), \\
& \operatorname{Hom}_{\mathrm{Tw}}\left(z^{\otimes m}, y^{\otimes n}\right) \cong \operatorname{Hom}_{C h}\left(Z_{b}^{\otimes m}, Y_{b}^{\otimes n}\right) \otimes P(m, n), \\
& \operatorname{Hom}_{\mathrm{Tw}}\left(x^{\otimes m}, z^{\otimes n}\right) \cong \operatorname{Hom}_{C h}\left(X_{b}^{\otimes m}, Z_{b}^{\otimes n}\right) \otimes P(m, n) .
\end{aligned}
$$

The method is exactly the same for the three cases, so we just write the argument for the third isomorphism. We need to determine the tensor powers of $z$. For every natural integer $n$, the object $z^{\otimes n}$ is given by the direct sum of shuffles

$$
\bigoplus_{\substack { 1 \leq j \leq i \leq n \\
\begin{subarray}{c}{\sigma \in \operatorname{Sh}(i, m-i) \\
\tau \in \operatorname{Sh}(j, m-j){ 1 \leq j \leq i \leq n \\
\begin{subarray} { c } { \sigma \in \operatorname { S h } ( i , m - i ) \\
\tau \in \operatorname { S h } ( j , m - j ) } }\end{subarray}} \sigma_{*}\left(\left(\mathbb{K} e_{0} \otimes x\right)^{\otimes n-i}, \tau_{*}\left(\left(\mathbb{K} e_{01} \otimes y\right)^{\otimes j},\left(\mathbb{K} e_{1} \otimes y\right)^{\otimes i-j}\right)\right),
$$

where the action $\sigma_{*}\left(A^{\otimes k}, B^{\otimes l}\right)$ of a $(k, l)$-shuffle $\sigma$ on a pair of tensor powers $\left(A^{\otimes k}, B^{\otimes l}\right)$ permutes the variables of the tensor product $A^{\otimes k} \otimes B^{\otimes l}$. The twisting of $z^{\otimes n}$ is determined by

$$
\mathrm{tw}_{0,01}^{\otimes n}=\mathrm{e}_{01}^{\otimes n} \otimes\left(\mathrm{e}_{0}^{\vee}\right)^{\otimes n} \otimes f^{\circ{ }_{h} n} \quad \text { and } \quad \mathrm{tw}_{1,01}^{\otimes n}=\mathrm{e}_{01}^{\otimes n} \otimes\left(\mathrm{e}_{1}^{\vee}\right)^{\otimes n} \otimes(-\mathrm{id})^{{ }^{\circ} h},
$$

where $o_{h}$ is the horizontal composition product of the dg prop $P_{x \rightarrow y}$. We get:

$$
\begin{aligned}
& \operatorname{Hom}_{\mathrm{Tw}}\left(x^{\otimes m}, z^{\otimes n}\right) \\
& =\bigoplus_{\substack{1 \leq j \leq i \leq n \\
\sigma \in \operatorname{Sh}(i, m-i) \\
\tau \in \operatorname{Sh}(j, m-j)}} \operatorname{Hom}_{\mathrm{T}_{\mathrm{w}}}\left(x^{\otimes m}, \sigma_{*}\left(\left(\mathbb{K} e_{0} \otimes x\right)^{\otimes n-i}, \tau_{*}\left(\left(\mathbb{K} e_{01} \otimes y\right)^{\otimes j},\left(\mathbb{K} e_{1} \otimes y\right)^{\otimes i-j}\right)\right)\right) \\
& \cong \bigoplus_{\substack{1 \leq j \leq i \leq n \\
\sigma \in \operatorname{Sh}(i, m-i) \\
\tau \in \operatorname{Sh}(j, m-j)}} \mathbb{K} e_{0}^{\otimes n-i} \otimes \mathbb{K} e_{01}^{\otimes j} \otimes \mathbb{K} e_{1}^{\otimes i-j} \otimes \operatorname{Hom}_{\mathrm{Tw}}\left(x^{\otimes m}, \sigma_{*}\left(x^{\otimes n-i}, \tau_{*}\left(y^{\otimes j}, y^{\otimes i-j}\right)\right)\right)
\end{aligned}
$$


Moreover, we have

$$
\begin{aligned}
\operatorname{Hom}_{\mathrm{Tw}}\left(x^{\otimes m}, \sigma_{*}\left(x^{\otimes n-i}, \tau_{*}\left(y^{\otimes j}, y^{\otimes i-j}\right)\right)\right) \\
\quad=P_{x \rightarrow y}\left(x, \ldots, x ; \sigma_{*}\left(x, \ldots, x, \tau_{*}(y, \ldots, y)\right)\right),
\end{aligned}
$$

where $P_{x \rightarrow y}\left(x, \ldots, x ; \sigma_{*}\left(x, \ldots, x, \tau_{*}(y, \ldots, y)\right)\right)$ has $m$ copies of the color $x$ as input, and as output $n-i$ copies of color $x$ and $i$ copies of color $y$ permuted by the shuffles $\sigma$ and $\tau$. We want to build an isomorphism

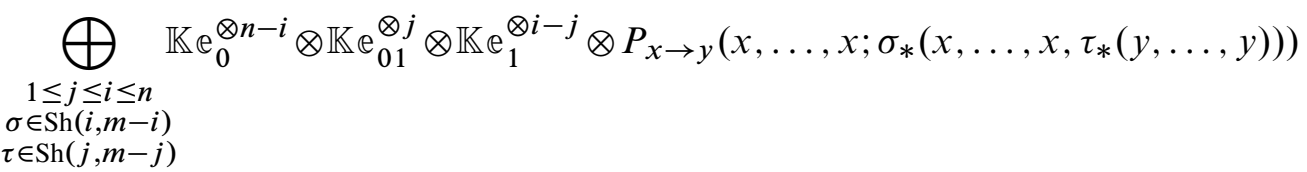

$$
\cong \bigoplus_{\substack{1 \leq j \leq i \leq n \\ \sigma \in \operatorname{Sh}(i, m-i) \\ \tau \in \operatorname{Sh}(j, m-j)}} \operatorname{Hom}_{\mathcal{C} h}\left(X_{b}^{\otimes m}, \sigma_{*}\left(X_{b}^{\otimes n-i}, \tau_{*}\left(Y_{b}[-1]^{\otimes j}, Y_{b}^{\otimes i-j}\right)\right)\right) \otimes P(m, n),
$$

where $[-1]$ is the degree shift applied to the chain complex $Y_{b}$. For this, we define in each component $(i, j, \sigma, \tau)$ of the direct sum an isomorphism

$$
\begin{aligned}
\mathbb{K} e_{0}^{\otimes n-i} \otimes \mathbb{K e}_{01}^{\otimes j} \otimes & \mathbb{K} e_{1}^{\otimes i-j} \otimes P_{x \rightarrow y}\left(x, \ldots, x ; \sigma_{*}\left(x, \ldots, x, \tau_{*}(y, \ldots, y)\right)\right) \\
& \rightarrow \operatorname{Hom}_{C h}\left(X_{b}^{\otimes m}, \sigma_{*}\left(X_{b}^{\otimes n-i}, \tau_{*}\left(Y_{b}[-1]^{\otimes j}, Y_{b}^{\otimes i-j}\right)\right)\right) \otimes P(m, n),
\end{aligned}
$$

which sends any

$$
\xi \in P_{x \rightarrow y}\left(x, \ldots, x ; \sigma_{*}\left(x, \ldots, x, \tau_{*}(y, \ldots, y)\right)\right)
$$

to

$$
\sigma_{*} \tau_{*} \otimes \sigma_{*}\left(f^{\circ{ }_{h} n-i}, \mathrm{id}^{\circ} h^{i}\right) \circ_{v} \xi
$$

where $\sigma_{*} \tau_{*}$ is the unique homomorphism sending $\mathbb{e}_{0}^{\otimes m}$ to $\sigma_{*}\left(\mathrm{e}_{0}^{\otimes n-i}, \tau_{*}\left(\mathrm{e}_{01}^{\otimes j}, \mathrm{e}_{1}^{\otimes i-j}\right)\right)$ and $\sigma_{*}\left(f^{\circ}{ }_{h} n-i, \mathrm{id}^{\circ}{ }^{\circ}\right)$ is the permutation of the variables in the iterated horizontal product $f \circ_{h} \cdots \circ_{h} f \circ_{h}$ id $\circ_{h} \cdots \circ_{h}$ id by $\sigma$.

Finally, since $\left(\left(i^{\otimes m}\right)^{*},\left(p^{\otimes n}\right)_{*}\right)$ is the tensor product of $\left(\left(i_{b}^{\otimes m}\right)^{*},\left(p_{b}^{\otimes n}\right)_{*}\right)$ by $P(m, n)$, it remains to apply the methods of [12, Lemma 8.3] in the category of chain complexes, for $X_{b}$ and $Y_{b}$, to prove that $\left(\left(i_{b}^{\otimes m}\right)^{*},\left(p_{b}^{\otimes n}\right)_{*}\right)$ is an acyclic fibration. We write the 
arguments here for the sake of clarity. We have the following commutative diagram:

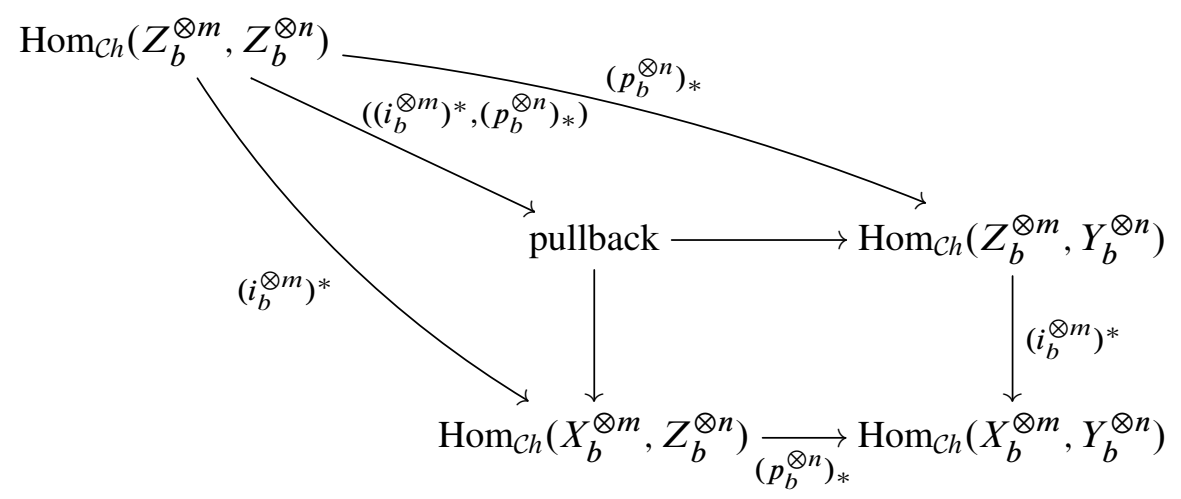

Recall that chain complexes over a field are all cofibrant and fibrant in the model structure of $\mathcal{C}$. The map $i_{b}$ is a cofibration and $X_{b}$ is cofibrant, so by the pushoutproduct axiom, for every integer $n$ the map $i_{b}^{\otimes n}: X^{\otimes n} \rightarrow Z^{\otimes n}$ is a cofibration. The category $\mathcal{C} h$ satisfies the limit monoid axioms [12, Section 6] and $Y_{b}$ is fibrant, so for every integer $n$ the map $p_{b}^{\otimes n}: Z_{b}^{\otimes n} \rightarrow Y_{b}^{\otimes n}$ is a fibration [12, Proposition 6.7]. Moreover, by the pushout-product axiom, the tensor product preserves acyclic cofibrations between cofibrant objects, so by Brown's lemma it preserves weak equivalences between cofibrant objects. Given that $Z_{b}$ and $Y_{b}$ are cofibrant, it implies that $p_{b}^{\otimes n}$ is an acyclic fibration. According to the dual pushout-product axiom, the fact that $i_{b}^{\otimes m}$ is a cofibration and $p_{b}^{\otimes n}$ is an acyclic fibration implies that $\left(\left(i_{b}^{\otimes m}\right)^{*},\left(p_{b}^{\otimes n}\right)_{*}\right)$ is an acyclic fibration.

\section{The subcategory of acyclic fibrations}

The goal of this section is to prove that the classifying space of weak equivalences of $P$-algebras is weakly equivalent to the classifying space of acyclic fibrations of $P$-algebras.

Theorem 3.1 Let $P$ be a cofibrant dg prop. The inclusion $i: f w \mathcal{C h}^{P} \hookrightarrow w_{C h} P$ of categories gives rise to a weak equivalence of simplicial sets, $\mathcal{N} f w \mathrm{Ch}^{P} \stackrel{\sim}{\rightarrow} \mathcal{N} w \mathrm{Ch}^{P}$.

Remark 3.2 Actually, the methods of [26] can be transposed in our setting to prove the following much stronger statement. We refer the reader to the seminal papers [10; 8; 9] for the notions of simplicial localization, hammock localization and Dwyer-Kan equivalences of simplicial categories. The inclusion of categories $i: f w \mathrm{Ch}^{P} \hookrightarrow w \mathrm{Ch}^{P}$ induces a Dwyer-Kan equivalence of hammock localizations

$$
L^{H}\left(\mathcal{C} h^{P}, f w C h^{P}\right) \stackrel{\sim}{\rightarrow} L^{H}\left(C h^{P}, w C h^{P}\right) .
$$


We refer the reader to [26] for more details about this proof, which relies on the properties of several models of $(\infty, 1)$-categories (simplicial categories [2], relative categories [1] and complete Segal spaces [22]).

To prove this theorem, we use Quillen's Theorem A [20]: we have to check that for every chain complex $X$, the nerve of the comma category $(X \downarrow i)$ is contractible. For this aim, we prove the following more general result.

Proposition 3.3 Let $\mathcal{I}$ be a small category. Every simplicial map $\mathcal{N} \mathcal{I} \rightarrow \mathcal{N}(X \downarrow i)$ is null up to homotopy.

As a consequence we get:

Proposition 3.4 The simplicial set $\mathcal{N}(X \downarrow i)$ is contractible.

To prove Proposition 3.4, we apply Proposition 3.3, for every $n \in \mathbb{N}$, to the subdivision category of a simplicial model of the $n$-sphere $S^{n}$. We take $\partial \Delta^{n+1}$ as simplicial model of $S^{n}$ and denote by sd $\partial \Delta^{n+1}$ its subdivision category. We then use general arguments of homotopical algebra.

Proposition 3.5 Let $F: \mathcal{C} \rightleftarrows \mathcal{D}: G$ be a Quillen adjunction. It induces natural isomorphisms

$$
\operatorname{Map}_{\mathcal{D}}(F(X), Y) \cong \operatorname{Map}_{\mathcal{C}}(X, G(Y))
$$

where $X$ is a cofibrant object of $\mathcal{C}$ and $Y$ a fibrant object of $\mathcal{D}$.

Proof We will use the definition of mapping spaces via cosimplicial frames. The proof works as well with simplicial frames. The adjunction $(F, G)$ induces an adjunction at the level of diagram categories

$$
F: \mathcal{C}^{\Delta} \rightleftarrows \mathcal{D}^{\Delta}: G
$$

Now let $\phi: A^{\bullet} \succ B^{\bullet}$ be a Reedy cofibration between Reedy cofibrant objects of $\mathcal{C}^{\Delta}$. This is equivalent, by definition, to saying that for every integer $r$, the maps

$$
(\lambda, \phi)_{r}: L^{r} B \coprod_{L^{r} A} A^{r} \succ B^{r}
$$

induced by $\phi$ and the latching object construction $L^{\bullet} A$ are cofibrations in $\mathcal{C}$. Let us consider the following pushout:

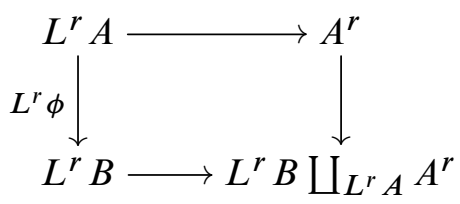


The fact that $\phi$ is a Reedy cofibration implies that for every $r$, the map $L^{r} \phi$ is a cofibration. Since cofibrations are stable under pushouts, the map $A^{r} \rightarrow L^{r} B \bigsqcup_{L^{r} A} A^{r}$ is also a cofibration. By assumption, the cosimplicial object $A^{\bullet}$ is Reedy cofibrant, so it is, in particular, pointwise cofibrant. We deduce that $L^{r} B \coprod_{L^{r} A} A^{r}$ is cofibrant. Similarly, each $B^{r}$ is cofibrant since $B^{\bullet}$ is Reedy cofibrant. The map $(\lambda, \phi)_{r}$ is a cofibration between cofibrant objects and $F$ is a left Quillen functor, so $F\left((\lambda, \phi)_{r}\right)$ is a cofibration of $\mathcal{D}$ between cofibrant objects. Recall that the $r^{\text {th }}$ latching object construction is defined by a colimit. As a left adjoint, the functor $F$ commutes with colimits so we get a cofibration

$$
L^{r} F\left(B^{\bullet}\right) \coprod_{L^{r}} \coprod_{F\left(A^{\bullet}\right)} F\left(A^{r}\right) \succ F\left(B^{r}\right)
$$

This means that $F(\phi)$ is a Reedy cofibration in $\mathcal{D}^{\Delta}$. Now, given that Reedy weak equivalences are the pointwise equivalences, if $\phi$ is a Reedy weak equivalence between Reedy cofibrant objects then it is, in particular, a pointwise weak equivalence between pointwise cofibrant objects, hence $F(\phi)$ is a Reedy weak equivalence in $\mathcal{D}^{\Delta}$. We conclude that $F$ induces a left Quillen functor between cosimplicial objects for the Reedy model structures. In particular, it sends any cosimplicial frame of a cofibrant object $X$ of $\mathcal{C}$ to a cosimplicial frame of $F(X)$.

Remark 3.6 The isomorphism above holds if the cosimplicial frame for the left-hand mapping space is chosen to be the image under $F$ of the cosimplicial frame of the right-hand mapping space. But recall that cosimplicial frames on a given object are all weakly equivalent, so that for any choice of cosimplicial frame we get at least weakly equivalent mapping spaces.

Now, recall that the geometric realization functor and the singular complex functor induce a Quillen equivalence

$$
|-|: \text { sSet } \rightleftarrows \text { Top :Sing. }(-)
$$

between topological spaces and simplicial sets. We have

$$
\begin{aligned}
\operatorname{Map}_{\mathrm{sSet}}\left(\mathcal{N} \operatorname{sd} \partial \Delta^{n+1}, \mathcal{N}(X \downarrow i)\right) & \simeq \operatorname{Map}_{\mathrm{sSet}}\left(\mathcal{N} \operatorname{sd} \partial \Delta^{n+1}, \operatorname{Sing}(|\mathcal{N}(X \downarrow i)|)\right) \\
& \simeq \operatorname{Map}_{\mathrm{Top}}\left(\left|\mathcal{N} \operatorname{sd} \partial \Delta^{n+1}\right|,|\mathcal{N}(X \downarrow i)|\right) \\
& \simeq \operatorname{Map}_{\mathrm{Top}}\left(S^{n},|\mathcal{N}(X \downarrow i)|\right)
\end{aligned}
$$

hence

$$
\left|\operatorname{Map}_{\mathrm{sSet}}\left(\mathcal{N} \operatorname{sd} \partial \Delta^{n+1}, \mathcal{N}(X \downarrow i)\right)\right| \simeq\left|\operatorname{Map}_{\mathrm{Top}}\left(S^{n},|\mathcal{N}(X \downarrow i)|\right)\right| ;
$$


in particular

$$
\pi_{0}\left|\operatorname{Map}_{\mathrm{sSet}}\left(\mathcal{N} \operatorname{sd} \partial \Delta^{n+1}, \mathcal{N}(X \downarrow i)\right)\right| \cong\left[S^{n},|\mathcal{N}(X \downarrow i)|\right]_{\mathrm{Ho}(\mathrm{Top})} .
$$

Proposition 3.3 means that for every integer $n$, the space

$$
\left|\operatorname{Map}_{\mathrm{sSet}}\left(\mathcal{N} \operatorname{sd} \partial \Delta^{n+1}, \mathcal{N}(X \downarrow i)\right)\right|
$$

has only one connected component (the component of the zero map); that is, the homotopy groups of $|\mathcal{N}(X \downarrow i)|$ are trivial.

Proof of Proposition 3.3 The category $(X \downarrow i)$ has weak equivalences $X \stackrel{\sim}{\rightarrow} Y$ as objects and acyclic fibrations as morphisms. It contains the initial object $X \stackrel{\equiv}{\rightarrow} X$ of $(X \downarrow C h)$.

Every simplicial map $\mathcal{N} \mathcal{I} \rightarrow \mathcal{N}(X \downarrow i)$ comes from a functor $\mathcal{I} \rightarrow(X \downarrow i)$, ie an $\mathcal{I}$ diagram in $(X \downarrow i)$. Let $F$ be such a functor. Let $\bar{X}$ be the initial $\mathcal{I}$-diagram, that is the constant diagram on $X \stackrel{\equiv}{\rightarrow} X$. In order to simplify notation, we write $Y$ for a morphism $X \rightarrow Y$ (an object of $(X \downarrow C h))$ and $Y \rightarrow Y^{\prime}$ for a commutative triangle relating $X \rightarrow Y$ to $X \rightarrow Y^{\prime}$ (a morphism of $(X \downarrow \mathcal{C} h)$ ). The diagram $F \times \bar{X}: \mathcal{I} \rightarrow(X \downarrow \mathcal{C} h)$ is defined on objects by $F \times \bar{X}(k)=F(k) \times X$ and on arrows by $F \times \bar{X}(\phi)=F(\phi) \times \operatorname{id}_{X}$. Applying the functorial factorization of Theorem 2.9 to the unique initial morphism $\bar{X} \rightarrow F \times \bar{X}$, we get a decomposition in $(X \downarrow C h)^{P}$ into a diagram $\mathcal{Y}$ given by

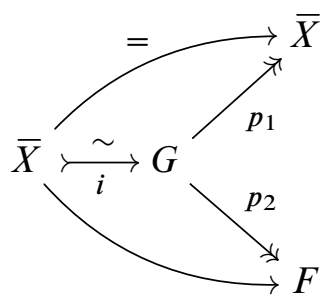

where the functor $G$ is defined pointwise by the functorial factorization of Theorem 2.9. The map $\left(p_{1}, p_{2}\right): G \rightarrow F \times \bar{X}$ is a pointwise fibration and $i$ is a pointwise acyclic cofibration of chain complexes. Since the map $\left(p_{1}, p_{2}\right): G \rightarrow F \times \bar{X}$ is a pointwise fibration and $F$ and $\bar{X}$ are pointwise fibrant, the maps $p_{1}$ and $p_{2}$ are pointwise acyclic fibrations: the product $F \times \bar{X}$ is given by the pullback

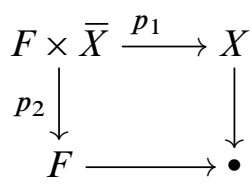

and pointwise fibrations are stable under pullbacks so $p_{1}$ and $p_{2}$ are pointwise fibrations. Since $\operatorname{id}_{\bar{X}}=p_{1} \circ i$ and $\bar{X} \rightarrow F=p_{2} \circ i$ are weak equivalences, the maps $p_{1}$ and $p_{2}$ are acyclic by the two-out-of-three property. 
The functors $\bar{X}$ and $F$ take their values in $(X \downarrow i)$ by definition. This implies that the functor $G$ sends morphisms of $\mathcal{I}$ to acyclic fibrations by definition of the functorial factorization in chain complexes. We consequently obtain a zigzag of natural transformations $\bar{X} \leftarrow G \rightarrow F$ of functors $\mathcal{I} \rightarrow(X \downarrow i)$. This zigzag implies that $\mathcal{N} F$ is homotopic to $\mathcal{N} \bar{X}$, which is itself null up to homotopy. This concludes the proof of Proposition 3.3.

\section{Moduli spaces of algebraic structures as homotopy fibers}

\subsection{Moduli spaces of algebra structures on fibrations}

The results of this subsection holds for algebras in $\mathcal{E}$ over a prop in $\mathcal{C}$, where the category $\mathcal{C}$ is a cofibrantly generated symmetric monoidal model category and the category $\mathcal{E}$ is a cofibrantly generated symmetric monoidal model category over $\mathcal{C}$. However, for the sake of simplicity we explain only the case $\mathcal{E}=\mathcal{C}=\mathcal{C} h$.

We start by recalling [12, Lemma 7.2]. Let $f: A \rightarrow B$ be a morphism of $\mathcal{C} h$. Then we have a pullback

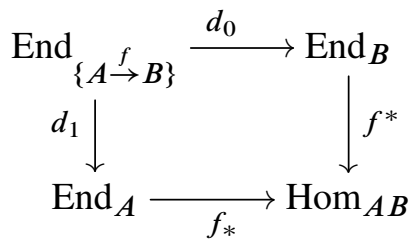

where $\operatorname{Hom}_{A B}$ is defined by $\operatorname{Hom}_{A B}(m, n)=\operatorname{Hom}_{C h}\left(A^{\otimes m}, B^{\otimes n}\right)$.

Lemma 4.1 [12, Lemma 7.2] (1) If $f$ is a (acyclic) fibration then so is $d_{0}$.

(2) If $f$ is a cofibration, then $d_{1}$ is a fibration. If $f$ is also acyclic then $d_{1}$ is an acyclic fibration and $d_{0}$ a weak equivalence.

Remark 4.2 Lemma 4.1 is a generalization in the prop context of [21, Propositions 4.1.7 and 4.1.8].

Lemma 4.3 Let $X_{n} \rightarrow \cdots \rightarrow X_{1} \rightarrow X_{0}$ be a chain of fibrations of chain complexes. For every $0 \leq k \leq n-1$, the map $d_{0}$ in the pullback

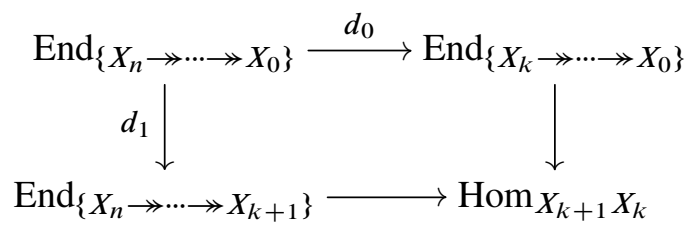


is a fibration. Moreover, if the fibrations in the chain $X_{n} \rightarrow \cdots \rightarrow X_{1} \rightarrow X_{0}$ are acyclic then so is $d_{0}$.

Proof We prove this lemma by induction. The case $n=1$ is Lemma 4.1. Now suppose that our lemma is true for a given integer $n \geq 1$. Let $X_{n+1} \rightarrow \cdots \rightarrow X_{1} \rightarrow X_{0}$ be a chain of fibrations of complexes. We distinguish two cases:

Case $\boldsymbol{k}=\boldsymbol{n}$ We have the pullback

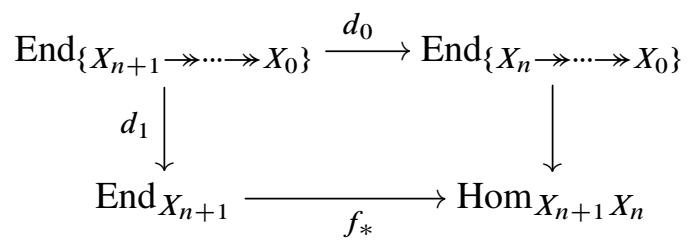

where $f: X_{n+1} \rightarrow X_{n}$. The fact that $f$ is a fibration implies that $f_{*}$ is a fibration, so $d_{0}$ is a fibration because of the stability of fibrations under pullback, and the acyclicity of $f$ implies the acyclicity of $d_{0}$. The detailed proof of these statements is done in the proof of [12, Lemma 7.2].

Case $0 \leq k \leq n-1$ We have that

$$
d_{0}=\operatorname{End}_{\left\{X_{n+1} \rightarrow \cdots \rightarrow X_{0}\right\}} \rightarrow \operatorname{End}_{\left\{X_{n} \rightarrow \cdots \rightarrow X_{0}\right\}} \rightarrow \operatorname{End}_{\left\{X_{k} \rightarrow \cdots \rightarrow X_{0}\right\}}
$$

is the composite of an map satisfying the induction hypothesis with the map of the case $k=n$, so the conclusion follows.

Remark 4.4 This lemma is the generalization of [21, Proposition 4.1.9] in the prop context.

We deduce from Lemmata 4.1 and 4.3 the following properties of our moduli spaces.

Proposition 4.5 Let $f: X \rightarrow Y$ be a chain complex morphism and $P$ be a cofibrant dg prop. The pullback of Lemma 4.1 gives rise to the following diagram of simplicial sets:

$$
P\{X\} \stackrel{\left(d_{1}\right)_{*}}{\longleftarrow} P\{f\} \stackrel{\left(d_{0}\right)_{*}}{\longrightarrow} P\{Y\}
$$

(1) If $f$ is a cofibration then $\left(d_{1}\right)_{*}$ is a fibration. Moreover, if $f$ is acyclic then $\left(d_{0}\right)_{*}$ and $\left(d_{1}\right)_{*}$ are weak equivalences.

(2) If $f$ is a fibration then $\left(d_{0}\right)_{*}$ is a fibration. Moreover, if $f$ is acyclic then $\left(d_{0}\right)_{*}$ and $\left(d_{1}\right)_{*}$ are weak equivalences. 
Proof (1) If $f$ is a cofibration then $d_{1}$ is a fibration. So $\left(d_{1}\right)_{*}$ is a fibration of simplicial sets according to Proposition 1.11. If $f$ is acyclic, then $d_{0}$ and $d_{1}$ are weak equivalences. Every chain complex is fibrant and cofibrant, and fibrations of props are determined componentwise, so $\operatorname{End}_{X}$ and $\mathrm{End}_{Y}$ are fibrant props. This implies that $\operatorname{End}_{\{f\}}$ is also fibrant. We deduce from this and Proposition 1.11 that $\left(d_{0}\right)_{*}$ and $\left(d_{1}\right)_{*}$ are weak equivalences.

(2) The proof is the same as in the previous case.

By induction we can also prove the following proposition.

Proposition 4.6 Let $X_{n} \stackrel{\sim}{\rightarrow} \cdots \stackrel{\sim}{\rightarrow} X_{1} \stackrel{\sim}{\rightarrow} X_{0}$ be a chain of acyclic fibrations and $P$ be a cofibrant dg prop. For every $0 \leq k \leq n-1$, the map $\left(d_{0}\right)_{*}$ is an acyclic fibration and $\left(d_{1}\right)_{*}$ a weak equivalence in the diagram below:

$$
P\left\{X_{n} \stackrel{\sim}{\rightarrow} \cdots \stackrel{\sim}{\rightarrow} X_{k+1}\right\} \stackrel{\left(d_{1}\right)_{*}}{\longleftarrow} P\left\{X_{n} \stackrel{\sim}{\rightarrow} \cdots \stackrel{\sim}{\rightarrow} X_{0}\right\} \stackrel{\left(d_{0}\right)_{*}}{\longrightarrow} P\left\{X_{k} \stackrel{\sim}{\rightarrow} \cdots \stackrel{\sim}{\rightarrow} X_{1}\right\}
$$

Remark 4.7 Propositions 4.5 and 4.6 are generalizations in the prop context of [21, Propositions 4.1.11, 4.1.12 and 4.1.13].

\subsection{Proof of Theorem 0.1}

We have now all the key results to generalize Rezk's theorem to algebras over dg props. The remaining arguments are the same as those of Rezk, so we will not repeat them with all details but essentially show how our Theorem 3.1, as well as the main theorem of [25], fit in the proof.

Let $P$ be a cofibrant dg prop, and $\mathcal{N} w C h^{P \otimes \Delta^{\bullet}}$ the bisimplicial set defined by

$$
\left(\mathcal{N} w \mathcal{C} h^{P \otimes \Delta^{\bullet}}\right)_{m, n}=\left(\left(\mathcal{N} w \mathcal{C} h^{c f}\right)^{P \otimes \Delta^{n}}\right)_{m} .
$$

The $\operatorname{dg}$ prop $P$ is cofibrant, thus so is $P \otimes \Delta^{n}$ for every $n \geq 0$. According to Theorem 3.1, we have a weak equivalence induced by an inclusion of categories

$$
\mathcal{N} f w C h^{P \otimes \Delta^{n}} \stackrel{\sim}{\rightarrow} \mathcal{N} w C h^{P \otimes \Delta^{n}} .
$$

Moreover, for every $n, n^{\prime} \geq 0$, the map $\Delta^{n} \rightarrow \Delta^{n^{\prime}}$ induces a weak equivalence of cofibrant dg props $P \otimes \Delta^{n} \rightarrow P \otimes \Delta^{n^{\prime}}$ and thereby a weak equivalence of simplicial sets

$$
\mathcal{N} w \mathcal{C} h^{P \otimes \Delta^{n^{\prime}}} \stackrel{\sim}{\rightarrow} \mathcal{N} w \mathcal{C} h^{P \otimes \Delta^{n}}
$$

according to [25, Theorem 0.1]. We obtain a zigzag of weak equivalences

$$
\operatorname{diag} \mathcal{N} f w C h^{P \otimes \Delta^{\bullet}} \stackrel{\sim}{\rightarrow} \operatorname{diag} \mathcal{N} w C h^{P \otimes \Delta^{\bullet}} \stackrel{\sim}{\leftarrow} w C h^{P} .
$$


We use an adaptation of a slightly modified version of Quillen's Theorem B (see [20]), namely [21, Lemma 4.2.2], in order to determine the homotopy fiber of the map $\operatorname{diag} \mathcal{N} f w \mathcal{C} h^{P \otimes \Delta^{\bullet}} \rightarrow \mathcal{N} f w \mathcal{C h}$. To prove that our map verifies the hypotheses of this lemma we use the propositions of Section 4.1 exactly in the same way as Rezk in the operadic case. Then we check that $\operatorname{diag}(U \downarrow X) \simeq P\{X\}$, where $U: f w \mathcal{C h}^{P \otimes \Delta^{\bullet}} \rightarrow$ $f w C h$ is the forgetful functor (by using again the propositions of Section 4.1) and finally we get the following diagram:

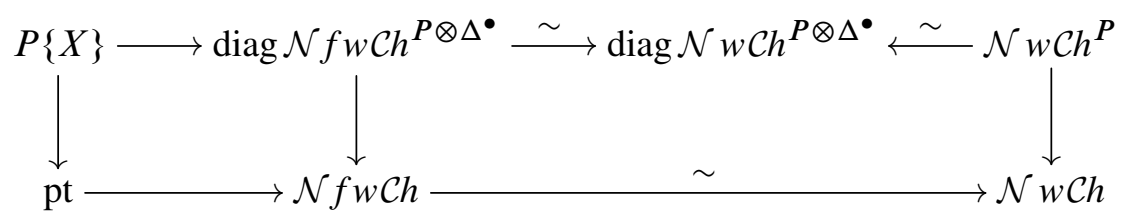

The proof of Theorem 0.1 is complete.

Remark 4.8 Note that we can recover the transfer theorem of bialgebra structures of [12, Theorem A] as a consequence of Theorem 0.1 . Indeed, let $P$ be a cofibrant dg prop in $\mathcal{C}$. Let $X \stackrel{\sim}{\rightarrow} Y$ be a morphism of $\mathcal{C}$ such that $Y$ is endowed with a $P$-algebra structure. We have a homotopy pullback of simplicial sets

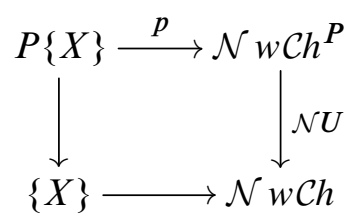

which induces an exact sequence of pointed sets

$$
\pi_{0} P\{X\} \rightarrow \pi_{0} \mathcal{N} w \mathcal{C} h^{P} \rightarrow \pi_{0} \mathcal{N} w \mathcal{C} h .
$$

The base point of the set $\pi_{0} \mathcal{N} w \mathcal{C} h$ is the weak equivalence class of $X$, denoted by $[X]$. The weak equivalence $X \stackrel{\sim}{\rightarrow} Y$ in $\mathcal{C} h$ implies that we have the equality $[Y]=[X]$ and thus $\pi_{0} \mathcal{N} U\left([Y]_{P}\right)=[X]$, where $[Y]_{P}$ is the weak equivalence class of $Y$ in $\mathrm{Ch}^{P}$. The exactness of the above sequence implies that $\pi_{0} p(P\{X\})=\left(\pi_{0} \mathcal{N} U\right)^{-1}([X])$ so $[Y]_{P} \in \pi_{0} p(P\{X\})$. This means that there exists a $P$-algebra structure on $X$ such that we have a zigzag of $P$-algebra morphisms

$$
X \stackrel{\sim}{\leftarrow} \stackrel{\sim}{\rightarrow} Y,
$$

which are weak equivalences of $\mathcal{C} h$.

Remark 4.9 We do not address the case of simplicial sets. However, [14, Theorem 1.4] endows the algebras over a colored prop in simplicial sets with a model category 
structure. Moreover, the free algebra functor exists in this case. Therefore one can transpose the methods used in the operadic setting to obtain a simplicial version of [25, Theorem 0.1]. Theorem 0.1 in simplicial sets can be proved by following Rezk's original proof step by step. We also conjecture that our results have a version in simplicial modules which would follow from arguments similar to ours.

\section{References}

[1] C Barwick, D M Kan, Relative categories: another model for the homotopy theory of homotopy theories, Indag. Math. 23 (2012) 42-68 MR2877401

[2] J E Bergner, A model category structure on the category of simplicial categories, Trans. Amer. Math. Soc. 359 (2007) 2043-2058 MR2276611

[3] M Chas, D Sullivan, String topology arXiv:math/9911159

[4] M Chas, D Sullivan, Closed string operators in topology leading to Lie bialgebras and higher string algebra, from "The legacy of Niels Henrik Abel” (O A Laudal, R Piene, editors), Springer, Berlin (2004) 771-784 MR2077595

[5] I Ciocan-Fontanine, M M Kapranov, Derived Hilbert schemes, J. Amer. Math. Soc. 15 (2002) 787-815 MR1915819

[6] V G Drinfel'd, Quantum groups, from "Proceedings of the International Congress of Mathematicians, I, II" (A M Gleason, editor), Amer. Math. Soc., Providence, RI (1987) 798-820 MR934283

[7] V G Drinfel'd, On quasitriangular quasi-Hopf algebras and on a group that is closely connected with $\mathrm{Gal}(\overline{\mathbf{Q}} / \mathbf{Q})$, Algebra i Analiz 2 (1990) 149-181 MR1080203 In Russian; translated in Leningrad Math. J. 2 (1991), 829-860

[8] W G Dwyer, D M Kan, Calculating simplicial localizations, J. Pure Appl. Algebra 18 (1980) 17-35 MR578563

[9] W G Dwyer, D M Kan, Function complexes in homotopical algebra, Topology 19 (1980) 427-440 MR584566

[10] W G Dwyer, D M Kan, Simplicial localizations of categories, J. Pure Appl. Algebra 17 (1980) 267-284 MR579087

[11] Y Frégier, M Markl, D Yau, The $L_{\infty}$-deformation complex of diagrams of algebras, New York J. Math. 15 (2009) 353-392 MR2530153

[12] B Fresse, Props in model categories and homotopy invariance of structures, Georgian Math. J. 17 (2010) 79-160 MR2640648

[13] P S Hirschhorn, Model categories and their localizations, Mathematical Surveys and Monographs 99, Amer. Math. Soc., Providence, RI (2003) MR1944041 
[14] M W Johnson, D Yau, On homotopy invariance for algebras over colored PROPs, J. Homotopy Relat. Struct. 4 (2009) 275-315 MR2559644

[15] G M Kelly, Basic concepts of enriched category theory, London Math. Soc. Lecture Note Series 64, Cambridge Univ. Press (1982) MR651714

[16] J Kock, Frobenius algebras and 2D topological quantum field theories, London Math. Soc. Student Texts 59, Cambridge Univ. Press (2004) MR2037238

[17] M Markl, Homotopy algebras are homotopy algebras, Forum Math. 16 (2004) 129-160 MR2034546

[18] M Markl, Intrinsic brackets and the $L_{\infty}$-deformation theory of bialgebras, J. Homotopy Relat. Struct. 5 (2010) 177-212 MR2812919

[19] S Merkulov, B Vallette, Deformation theory of representations of prop(erad)s, II, J. Reine Angew. Math. 636 (2009) 123-174 MR2572248

[20] D Quillen, Higher algebraic $K$-theory, $I$, from "Algebraic $K$-theory, I: Higher $K-$ theories" (H Bass, editor), Lecture Notes in Math. 341, Springer, Berlin (1973) 85-147 MR0338129

[21] C W Rezk, Spaces of algebra structures and cohomology of operads, $\mathrm{PhD}$ thesis, Massachusetts Institute of Technology (1996) MR2716655 Available at http:// search.proquest.com/docview/304311860

[22] C Rezk, A model for the homotopy theory of homotopy theory, Trans. Amer. Math. Soc. 353 (2001) 973-1007 MR1804411

[23] M Schlessinger, J Stasheff, The Lie algebra structure of tangent cohomology and deformation theory, J. Pure Appl. Algebra 38 (1985) 313-322 MR814187

[24] V G Turaev, Skein quantization of Poisson algebras of loops on surfaces, Ann. Sci. École Norm. Sup. 24 (1991) 635-704 MR1142906

[25] S Yalin, Classifying spaces of algebras over a prop, Algebr. Geom. Topol. 14 (2014) 2561-2593 MR3276841

[26] S Yalin, Simplicial localisation of homotopy algebras over a prop, Math. Proc. Cambridge Philos. Soc. 157 (2014) 457-468 MR3286518

[27] S Yalin, Moduli stacks of algebraic structures and deformation theory, J. Noncommut. Geom. 10 (2016) 579-661 MR3519047

Department of Mathematical Sciences, University of Copenhagen

Universitetsparken 5, 2100 Copenhagen, Denmark

yalinprop@gmail.com

Received: 5 February 2015 Revised: 26 February 2016 
